\title{
Volatile snowlines in embedded disks around low-mass protostars $^{\star}$
}

\author{
D. Harsono ${ }^{1,2,4}$, S. Bruderer ${ }^{3}$, and E. F. van Dishoeck ${ }^{1,3}$ \\ ${ }^{1}$ Leiden Observatory, Leiden University, Niels Bohrweg 2, 2300 RA Leiden, The Netherlands \\ e-mail: harsono@strw.leidenuniv.nl \\ 2 SRON Netherlands Institute for Space Research, PO Box 800, 9700 AV Groningen, The Netherlands \\ 3 Max-Planck-Institut für extraterretrische Physik, Giessenbachstrasse 1, 85748 Garching, Germany \\ ${ }^{4}$ Heidelberg University, Center for Astronomy, Institute of Theoretical Astrophysics, Albert-Ueberle-Straße 2, \\ 69120 Heidelberg, Germany
}

Received 25 February 2015 / Accepted 27 July 2015

\begin{abstract}
Context. Models of the young solar nebula assume a hot initial disk in which most volatiles are in the gas phase. Water emission arising from within $50 \mathrm{AU}$ radius has been detected around low-mass embedded young stellar objects. The question remains whether an actively accreting disk is warm enough to have gas-phase water up to $50 \mathrm{AU}$ radius. No detailed studies have yet been performed on the extent of snowlines in an accreting disk embedded in a dense envelope (stage 0 ).

Aims. We aim to quantify the location of gas-phase volatiles in the inner envelope and disk system for an actively accreting embedded disk.

Methods. Two-dimensional physical and radiative transfer models were used to calculate the temperature structure of embedded protostellar systems. Heating due to viscous accretion was added through the diffusion approximation. Gas and ice abundances of $\mathrm{H}_{2} \mathrm{O}, \mathrm{CO}_{2}$, and $\mathrm{CO}$ were calculated using the density-dependent thermal desorption formulation.

Results. The midplane water snowline increases from 3 to $\sim 55$ AU for accretion rates through the disk onto the star between $10^{-9}-$ $10^{-4} M_{\odot} \mathrm{yr}^{-1} . \mathrm{CO}_{2}$ can remain in the solid phase within the disk for $\dot{M} \leq 10^{-5} M_{\odot} \mathrm{yr}^{-1}$ down to $\sim 20$ AU. Most of the CO is in the gas phase within an actively accreting disk independent of disk properties and accretion rate. The predicted optically thin water isotopolog emission is consistent with the detected $\mathrm{H}_{2}^{18} \mathrm{O}$ emission toward the stage 0 embedded young stellar objects, originating from both the disk and the warm inner envelope (hot core). An accreting embedded disk can only account for water emission arising from $R<50 \mathrm{AU}$, however, and the extent rapidly decreases for $\dot{M} \leq 10^{-5} M_{\odot} \mathrm{yr}^{-1}$. Thus, the radial extent of the emission can be measured with future ALMA observations and compared to this 50 AU limit.

Conclusions. Volatiles such as $\mathrm{H}_{2} \mathrm{O}, \mathrm{CO}_{2}, \mathrm{CO}$, and the associated complex organics sublimate out to $50 \mathrm{AU}$ in the midplane of young disks and, thus, can reset the chemical content inherited from the envelope in periods of high accretion rates $\left(>10^{-5} M_{\odot} \mathrm{yr}^{-1}\right)$. A hot young solar nebula out to $30 \mathrm{AU}$ can only have occurred during the deeply embedded stage 0 , not during the T Tauri phase of our early solar system.
\end{abstract}

Key words. stars: formation - ISM: molecules - accretion, accretion disks - astrochemistry - stars: low-mass - stars: protostars

\section{Introduction}

The snowlines of various volatiles (sublimation temperature $T_{\text {sub }} \lesssim 160 \mathrm{~K}$ ) play a major role for planet formation. Beyond the snowline, the high abundances of solids allow for efficient sticking to form larger bodies, which is further enhanced by the presence of ices (e.g., Stevenson \& Lunine 1988; Ros \& Johansen 2013). Extensive studies have investigated the snowline in protoplanetary disks around pre-main-sequence stars similar to the nebula out of which the solar system is assumed to have formed (e.g., Lissauer 1987; Pollack et al. 1996). In such models, the water snowline is located at a radius of a few AU. It is thought that the early pre-solar nebula was hot $(>1500 \mathrm{~K})$, such that both volatiles and refractories $\left(T_{\text {sub }} \gtrsim 1400 \mathrm{~K}\right)$ are in the gas phase out to larger distances (Cassen 2001; Scott 2007; Davis et al. 2014; Marboeuf et al. 2014). The evidence of such a hot solar nebula comes from the history of the refractories, but the volatile content of comets seems to indicate that a part of the disk remains

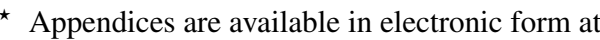
http://www. aanda.org
}

cold (Bockelée-Morvan et al. 2000; Mumma \& Charnley 2011; Pontoppidan et al. 2014). The evolution of the snowline due to disk and star evolution and its accretion rate clearly affects the chemical composition in the region relevant to planet formation (e.g., Lodders 2004; Davis 2005; Öberg et al. 2011b). The most relevant volatiles are the known major ice species: $\mathrm{H}_{2} \mathrm{O}, \mathrm{CO}_{2}$, and CO. Observations (Meijerink et al. 2009; Zhang et al. 2013) and models (e.g., D'Alessio et al. 1998; Dullemond et al. 2007) of protoplanetary disks around pre-main sequence $\mathrm{T}$ Tauri stars indicate that such disks are not warm enough to have gas-phase volatiles in the midplane beyond $30 \mathrm{AU}$, as claimed in some early solar nebula models, and, for the case of $\mathrm{H}_{2} \mathrm{O}$, a snowline of only a few AU is typically found. Higher temperatures at large radii might be achieved, but only during the deeply embedded phase of star formation when the accretion rate is high. The question remains how hot an embedded accreting disk can be when the accretion rate is high $\left(\geq 10^{-6} M_{\odot} \mathrm{yr}^{-1}\right.$, see Dunham et al. 2014, for a recent review).

Significant progress has been made in identifying snowlines in protoplanetary disks in the later stages when the envelope has 
dissipated and on their location with respect to gas giant formation sites (e.g., Kennedy \& Kenyon 2008; Pontoppidan et al. 2014). Direct observational evidence of snowlines of the major ice species toward protoplanetary disks around pre-main sequence stars relies on the chemical changes that occur when a molecule is absent in the gas phase. The most readily observed snowline is that of $\mathrm{CO}$ as inferred through spatially resolved observations of $\mathrm{N}_{2} \mathrm{H}^{+}$, whose gas-phase abundance is enhanced when CO is frozen-out (e.g., Qi et al. 2013). $\mathrm{DCO}^{+}$ is also a tracer of cold gas at temperatures close to that of the CO snowline (van Dishoeck et al. 2003; Guilloteau et al. 2006; Qi et al. 2008; Mathews et al. 2013). Both tracers indicate $\mathrm{CO}$ snowlines at $>30 \mathrm{AU}$ for $\mathrm{T}$ Tauri disks to $>100 \mathrm{AU}$ for disks around Herbig stars. The water snowline has been inferred to be within a few AU from direct observation and modeling of midinfrared water lines (Meijerink et al. 2009; Zhang et al. 2013). The $\mathrm{CO}$ snowline location with respect to those of water and $\mathrm{CO}_{2}$ has a direct impact on the amount of water present in giant planet atmospheres (Öberg et al. 2011b; Madhusudhan et al. 2011; Moses et al. 2013).

Snowlines in the early embedded stages of star formation can be much farther out, however, because the stellar accretion process through the disk onto the star begins at the time that the disk itself is forming. The gaseous volatile reservoir in embedded disks is affected by their formation process, which results in emerging protoplanetary disks having different chemical structures depending on initial cloud core parameters (Visser et al. 2011). A related question centers on whether these volatiles are "inherited" or "reset" during the planet formation process (Pontoppidan et al. 2014). The reset scenario refers to the chemical processing of ices as the gas and dust are exposed to elevated temperatures $(>40 \mathrm{~K})$ during their voyage from the envelope to the disk. These temperature regions define the regions where both $\mathrm{CO}_{2}$ and $\mathrm{CO}$ sublimate from the ice in the gas phase. Both species are difficult to trace in embedded disks, however: $\mathrm{CO}_{2}$ because it lacks a dipole moment, and $\mathrm{CO}$ because of confusion with the surrounding envelope.

The effect of accretion may be most readily seen through the changes in the water snowline. Water is the major constituent of ices on the grains that facilitate planet formation (Gibb et al. 2004; Öberg et al. 2011a) and a major coolant (Karska et al. 2013), and thus a key volatile in star- and planet formation. Most of the water is thought to be formed during the pre-stellar stage and then transported through the envelope into the disk and planets (Visser et al. 2009; Cleeves et al. 2014; van Dishoeck et al. 2014). The crucial step that is relatively unexplored as yet is the processing of water during disk formation.

The water vapor content around protostars is investigated by the "Water In Star-forming regions with Herschel" key program (WISH, van Dishoeck et al. 2011). As a result of the large beam of Herschel, a significant fraction of the detected water emission stems from the large-scale envelope and the bipolar outflow (Kristensen et al. 2012; Herczeg et al. 2012; Mottram et al. 2014). More importantly, the outflowing water will escape the system and will not be retained by the disk. To determine the amount of water vapor associated with the inner envelope or disk, an isotopolog of warm water $\left(\mathrm{H}_{2}^{18} \mathrm{O}\right)$ needs to be observed. Visser et al. (2013) reported a detection of the $\mathrm{H}_{2}^{18} \mathrm{O}$ line (1096 GHz $E_{\mathrm{u}}=249 \mathrm{~K}$ ) with HIFI (de Graauw et al. 2010) toward the embedded protostar NGC 1333 IRAS 2A. However, they found that the line is still optically thick with an emitting region of $\sim 100 \mathrm{AU}$. Thus, it remains difficult to constrain the amount of water vapor in embedded disks through single-dish observations.
Spatially resolved warm $\mathrm{H}_{2}^{18} \mathrm{O}$ emission (excitation temperature $T_{\mathrm{ex}} \sim 120 \mathrm{~K}$ Persson et al. 2014) has recently been detected from within the inner $50 \mathrm{AU}$ radius of several deeply embedded (class 0) low-mass protostars (e.g., Jørgensen \& van Dishoeck 2010; Persson et al. 2012, 2013) and toward one high-mass disk (van der Tak et al. 2006; Wang et al. 2012). The low-mass sources are very young objects whose envelope mass is substantially higher than the mass at small scales $(R \lesssim 100 \mathrm{AU}$; also denoted as stage 0, Robitaille et al. 2006). The emission is expected to arise from $T_{\text {dust }}>100 \mathrm{~K}$ regions where ice sublimates and the gas-phase water abundance is at its maximum (Fraser et al. 2001; Aikawa et al. 2008; Mottram et al. 2013). This inner region is, however, also where the disk forms (Larson 2003; Williams \& Cieza 2011; Li et al. 2014). Rotationally supported disks have recently been detected around a few low-mass protostars (e.g., Tobin et al. 2012; Murillo et al. 2013; Ohashi et al. 2014). Based on the kinematical information, both Jørgensen \& van Dishoeck (2010) and Persson et al. (2012) found that the water emission does not show Keplerian motion and concluded that it must be emitted from a flattened disk-like structure that is still dominated by the radial infalling motions.

This paper investigates snowlines of volatiles within an accreting disk embedded in a massive envelope. The spatial extent and the water vapor emission are compared with the observed values toward three deeply embedded low-mass protostars. The thermal structure of an actively accreting disk is computed including the additional heating that is due to the energy released from the viscous dissipation. Most previous studies of the thermal structure of an accreting disk focused on the later evolutionary stage of disk evolution where the envelope has largely dissipated away. Furthermore, they focused on the midplane temperature structure (e.g., Sasselov \& Lecar 2000; Lecar et al. 2006; Kennedy \& Kenyon 2008). The additional heating in the embedded phase shifts the snowlines of volatiles outward to larger radii than in disks around pre-main sequence stars (Davis 2005; Garaud \& Lin 2007; Min et al. 2011). The details of the physical and chemical structure of the embedded disk are presented in Sect. 2. Section 3 presents the snowline locations as function of disk and stellar properties. The results are compared with observations, and their implications on the young solar nebula is discussed in Sect. 4. Section 5 summarizes the main results and conclusions.

\section{Physical and chemical structures}

\subsection{Physical structure}

A parametrized embedded disk (disk + flattened envelope) model was used to construct the density structure following Crapsi et al. (2008). The main parameters are disk mass $\left(M_{\text {disk }}\right)$ and disk radius $\left(R_{\text {disk }}\right)$. A number of parameters defining the envelope and the disk are fixed and summarized in Table 1. The envelope mass was fixed at $1 M_{\odot}$, which is appropriate for the objects from which the water emission has been detected (Jørgensen et al. 2009; Kristensen et al. 2012, and see Sect. 4.3). The mass distribution within a flattened envelope is more crucial for the temperature structure than the total mass.

For the large-scale envelope, a flattened envelope due to rotation as described by Ulrich (1976) was adopted, whose densities are given by the following equation:

$\rho_{\mathrm{env}}(r, \mu) \propto\left(\frac{R_{\mathrm{cen}}}{r}\right)^{1.5}\left(1+\frac{\mu}{\mu_{0}}\right)^{-1 / 2}\left(\frac{\mu}{2 \mu_{0}}+\frac{R_{\mathrm{cen}}}{r} \mu_{0}^{2}\right)^{-1}$, 
Table 1. Parameters for the embedded disk + envelope models.

\begin{tabular}{llc}
\hline \hline Variable [unit] & Description & Value(s) \\
\hline$r_{\text {out }}[\mathrm{AU}]$ & Outer radius & $10^{4}$ \\
$r_{\text {in }}[\mathrm{AU}]$ & Inner radius & 0.1 \\
$R_{\text {cen }}[\mathrm{AU}]$ & Centrifugal radius & $50, \mathbf{2 0 0}$ \\
$M_{\text {env }}\left[M_{\odot}\right]$ & Envelope mass & 1.0 \\
$M_{\text {disk }}\left[M_{\odot}\right]$ & Disk mass & $0.05, \mathbf{0 . 1}, 0.2,0.5$ \\
$R_{\text {disk }}[\mathrm{AU}]$ & Disk radii & $50, \mathbf{1 0 0}, 200$ \\
$H_{0}[\mathrm{AU}]$ & Scale height at $1 \mathrm{AU}$ & 0.2 \\
$T_{\star}[\mathrm{K}]$ & Stellar temperature & 4000 \\
$M_{\star}\left[M_{\odot}\right]$ & Stellar mass & 0.5 \\
$L_{\star}\left[L_{\odot}\right]$ & Stellar luminosity & $\mathbf{1}, 5,15$ \\
$\dot{M}\left[M_{\odot} \mathrm{yr}^{-1}\right]$ & Accretion rate & $10^{-4,-4.2,-4.5,-5,-6,-7,-9}$ \\
\hline
\end{tabular}

Notes. The varied parameters and the canonical values are indicated in boldface.

where $\mu \equiv \cos \theta, R_{\text {cen }}$ the centrifugal radius, and $r$ the spherical radius. The centrifugal radius defines the region in which the material no longer flows radially and enters the disk. Two centrifugal radii of $R_{\text {cen }}=50 \mathrm{AU}$ and $200 \mathrm{AU}$ were explored. The low $R_{\text {cen }}$ corresponds to the small Keplerian disk toward L1527 (Ohashi et al. 2014), while the high $R_{\text {cen }}$ corresponds to the maximum disk radius ( $\sim 180 \mathrm{AU})$ observed toward a class 0 embedded low-mass young stellar objects (YSO; Murillo et al. 2013). The two cases explore the effect of mass concentration in the inner envelope on water emission. For a given centrifugal radius, a particle follows a parabolic motion given by

$\frac{r}{R_{\text {cen }}} \frac{1-\mu / \mu_{0}}{1-\mu_{0}^{2}}=1$,

where $\mu_{0}$ satisfies the condition above at every $r$ and $\mu$. The outer radius of the envelope is fixed at $r_{\text {out }}=10^{4} \mathrm{AU}$ with an inner radius of $0.1 \mathrm{AU}$, where the dust typically sublimates (assuming a dust sublimation temperature, $T_{\text {sub }}$, between 1500-2000 K).

An outflow cavity is then carved out from the envelope density structure at $\mu_{0}>0.95$. Following Crapsi et al. (2008), the density inside the cavity is equal to that of the densities at $r_{\text {out }}$. This creates a conical outflow with an aperture of $30^{\circ}$ at large radii (semi-aperture of $15^{\circ}$ ). For a $1 M_{\odot}$ envelope, gas densities of $\sim 10^{4} \mathrm{~cm}^{-3}$ fill the envelope cavity, which is consistent with those observed toward YSOs (e.g., Bachiller \& Tafalla 1999; Whitney et al. 2003).

A flared accretion disk is added to the envelope density structure. The density within the disk follows the power-law dependence radially and has a Gaussian distribution vertically in $z$ as expected from a hydrostatic disk. The flared disk densities (Shakura \& Sunyaev 1973; Pringle 1981; Hartmann et al. 1998; Williams \& Cieza 2011) are described by

$\rho_{\text {disk }}(R, z)=\frac{\Sigma_{0} \times\left(R / R_{\text {disk }}\right)^{-1}}{\sqrt{2 \pi} H(R)} \exp \left[-\frac{1}{2}\left(\frac{z}{H(R)}\right)^{2}\right]$,

where the scale height $H$ is fixed to $0.2 \mathrm{AU}\left(H_{0}\right)$ at $1 \mathrm{AU}\left(R_{0}\right)$, $R_{\text {disk }}$ the disk radius, and $R$ the cylindrical radius. The radial dependence of the scale height is $H(R)=R H_{0} / R_{0}\left(R / R_{0}\right)^{2 / 7}$ (Chiang \& Goldreich 1997). Finally, the densities are scaled by a constant factor $\Sigma_{0}$ such that the total disk mass within $R_{\text {disk }}$ $\left(R<R_{\text {disk }}\right)$ is equal to the values in Table 1 and distributed within $R_{\text {disk }}$. In the case of $R_{\text {cen }}=50$ AU flattened envelopes, only $R_{\text {disk }}=50 \mathrm{AU}$ models are considered. The total gas density in the model is $\rho=\rho_{\text {disk }}+\rho_{\text {env }}$ with a gas-to-dust mass ratio of 100 .

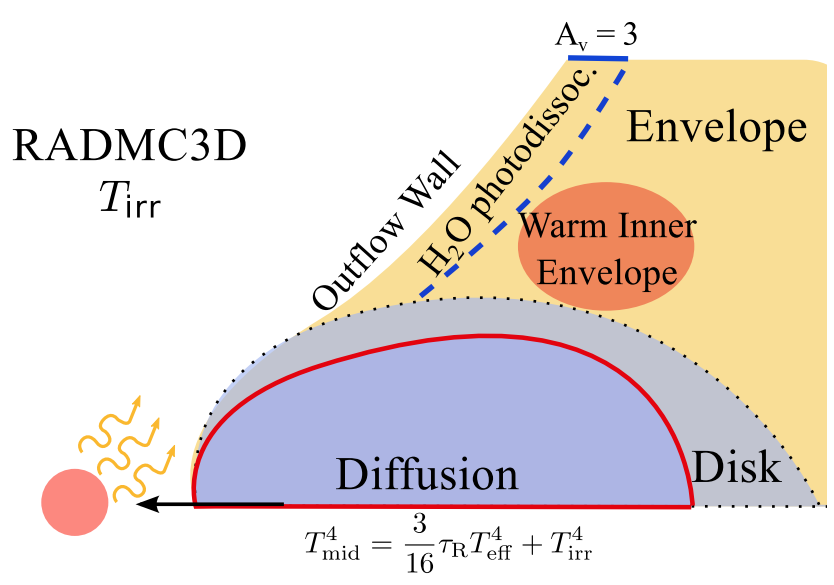

Fig. 1. Schematic showing the calculation of the thermal structure of the disk by combining both the RADMC3D Monte Carlo simulation and the diffusion equation. The Monte Carlo simulation calculates the temperature structure due to the irradiation $\left(T_{\text {irr }}\right)$ from a central star, while the diffusion equation is used to solve the temperature structure at high optical depths $\left(\tau_{\mathrm{R}}>1\right.$ where $\tau_{\mathrm{R}}$ is the Rosseland mean optical depth) as indicated by the red line. This high optical depth region typically starts below the disk surface. The outflow wall, warm inner envelope $\left(T_{\text {dust }}>100 \mathrm{~K}\right.$ and $\left.r<500 \mathrm{AU}\right)$, and photodissociation region are indicated.

\subsection{Temperature structure and heating terms}

The three-dimensional dust continuum radiative transfer code RADMC- $3 \mathrm{D}^{1}$ was used to calculate the dust temperature structure. A central star with temperature of $4000 \mathrm{~K}$ (a typically inferred effective temperature of class I protostars; White \& Hillenbrand 2004; Nisini et al. 2005) characterized by $L_{\star} 1,5$, and $15 L_{\odot}$ was adopted. An accurate dust temperature structure of the disk is crucial in determining the location where the various volatiles thermally desorb from the grain. This is computationally challenging for a massive optically thick disk as we modeled here (see Min et al. 2009). Thus, we separated the dust temperature calculations due to the central star irradiation (passive) from the viscous heating treatment (see Fig. 1). The former was determined by RADMC3D considering a black body central star as listed in Table 1.

An actively accreting disk provides additional heating $\left(L_{\text {disk }}\right)$ from the loss of mechanical energy as the gas is viscously transported inward. The steady-state accretion rate is typically between $10^{-5}-10^{-7} M_{\odot} \mathrm{yr}^{-1}$ (Hueso \& Guillot 2005). However, episodic accretion events such as those simulated by Vorobyov (2009) can have transient spikes with an accretion rate of up to $10^{-4} M_{\odot} \mathrm{yr}^{-1}$. Thus, stellar accretion rates between $10^{-9}-$ $10^{-4} M_{\odot} \mathrm{yr}^{-1}$ were adopted, following the $\alpha$ disk formalism (Shakura \& Sunyaev 1973; Williams \& Cieza 2011). The viscous heating rate per volume is given by

$Q_{\mathrm{visc}}=\frac{9}{4} \rho_{\mathrm{disk}} \nu \Omega^{2}$,

where $v=\alpha c_{\mathrm{s}} H$ is the $\alpha$-dependent turbulent viscosity parameter, $c_{\mathrm{s}}$ the sound speed of the gas, and $\Omega$ the Keplerian angular velocity. At steady state, the viscosity is related to the disk mass and the accretion rate through (Lodato 2008)

$\dot{M}=3 \pi v \Sigma$.

\footnotetext{
1 http://www.ita.uni-heidelberg.de/ dullemond/ software/radmc-3d
} 


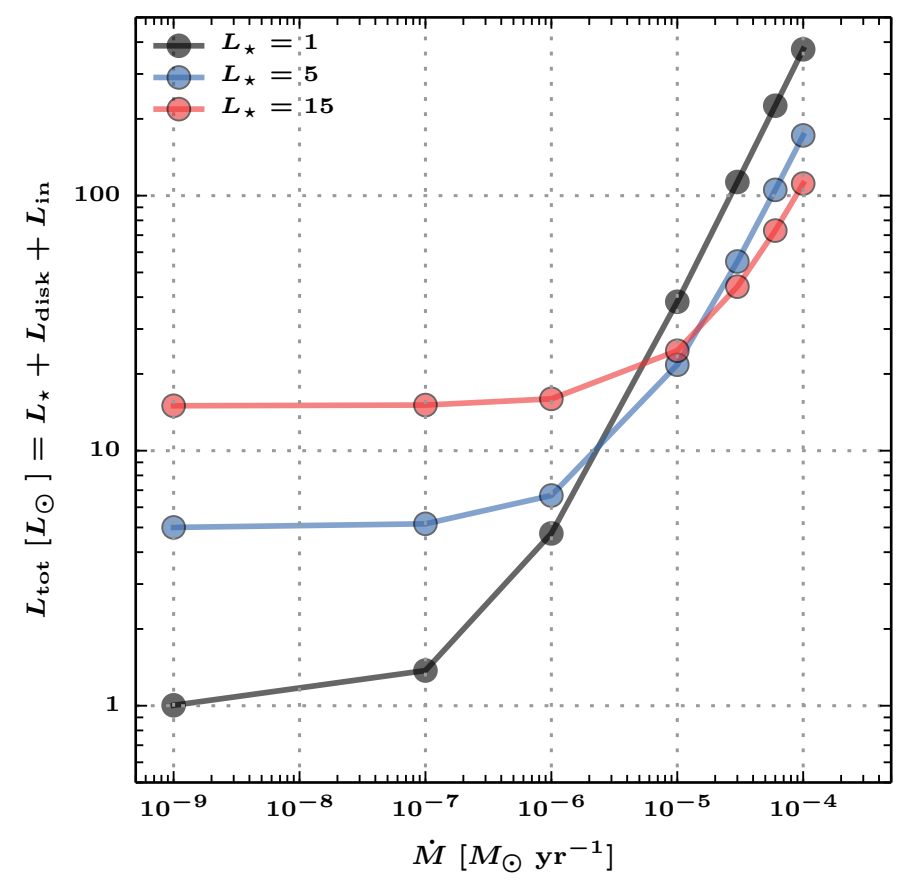

Fig. 2. Total luminosity $\left(L_{\mathrm{tot}}=L_{\star}+L_{\mathrm{disk}}+L_{\mathrm{in}}\right)$ as a function of accretion rates for different stellar luminosities.

To explore the degree of heating, the viscosity term $v$ was varied for the explored accretion rates at a fixed disk mass $(\propto \Sigma)$. The effective temperature $\left(T_{\text {visc }}\right)$ associated with the energy released at the inner radius assuming a hydrostatic disk is

$\sigma_{\mathrm{SB}} T_{\mathrm{visc}}^{4}(R)=\int Q_{\mathrm{visc}}(R) \mathrm{d} z=\frac{3}{8 \pi} \frac{G M_{\star}}{R^{3}} \dot{M}\left(1-\sqrt{\frac{R_{\star}}{R}}\right)$.

This was obtained by integrating the viscous heating terms vertically at all radii. Furthermore, $T_{\text {visc }}$ is the effective temperature of the disk at the optically thin photosphere without the addition of stellar irradiation. However, the midplane temperature of an active disk is proportional to the vertical optical depth (Hubeny 1990): $T_{\text {mid }}^{4} \sim \kappa_{\mathrm{R}} \Sigma_{\text {gas }} T_{\text {visc }}^{4}$ with $\kappa_{R}$ the Rosseland mean opacity which results in higher midplane temperatures. This method is similar to that of Kennedy \& Kenyon (2008) and Hueso \& Guillot (2005) in the optically thick regime. We note that the heating from an accreting disk is caused by the dissipation of energy from both gas and dust. To account for the irradiation from the accreting disk, $L_{\mathrm{disk}}=\int \pi \sigma T_{\mathrm{visc}}(R)^{4} R \mathrm{~d} R$ was added to the central luminosity $L_{\star}$ by determining its black body spectrum at $T_{\text {visc }}$ at all radii of the disk. For the case of high accretion rates, it is expected that the accretion proceeds onto the star since a gaseous disk is present within the dust sublimation radius. The total luminosity from the inner disk is $L_{\text {in }}=\int_{\mathrm{R}_{\text {in }}}^{\mathrm{R}_{\star}} \pi \sigma T_{\mathrm{visc}}^{4} R \mathrm{~d} R$. Thus, the final irradiating central source $L_{\text {tot }}$ is the combined heating from the central star, the dusty disk, and the inner gaseous disk: $L_{\mathrm{tot}}=L_{\star}+L_{\mathrm{disk}}+L_{\mathrm{in}}$. Figure 2 shows the relation between $L_{\text {tot }}$ and $\dot{M}$ for different $L_{\star}$ values.

The following steps were taken to calculate the dust temperature of an accreting embedded disk.

- Monte Carlo dust continuum radiative transfer was used to simulate the photon propagation to determine the passively heated dust temperature structure $\left(T_{\text {irr }}\right)$ due to total luminosity $\left(L_{\mathrm{tot}}\right)$. The dust opacities of Crapsi et al. (2008) were adopted; they are composed of a distribution of ice-coated silicates and graphite grains. The removal of ices from the grain at $T_{\text {dust }}>100 \mathrm{~K}$ does not strongly alter the dust temperature structure.

- Viscous heating was added to the region of the disk where the Rosseland mean optical depth $\tau_{\mathrm{R}}>1$. This was made by fixing the midplane temperatures to $T_{\text {mid }}=\left(\frac{3}{16} \kappa_{\mathrm{R}} \Sigma_{\text {gas }} T_{\text {visc }}^{4}+T_{\text {irr }}^{4}\right)^{1 / 4}$. The vertical dust temperature structure was calculated using the diffusion approximation $\nabla D \nabla T^{4}=0$ bounded by $T_{\text {irr }}$ at the surface as obtained from the previous step and $T_{\text {mid }}$ at the midplane where $D=\left(3 \rho_{\text {dust }} \kappa_{\mathrm{R}}\right)^{-1}$. The diffusion was performed only within the $\tau_{\mathrm{R}}>1$ regions. This calculation was repeated a few times until it converged. Convergence was obtained when there was no longer any change in the the $\tau_{\mathrm{R}}>1$ region.

The addition of the viscous heating can increase the dust temperatures to $>2000 \mathrm{~K}$, while the typical dust vaporization temperature is $\sim 1500 \mathrm{~K}$. Therefore, we used an upper limit to the dust temperature of $1500 \mathrm{~K}$. Gas opacities in the inner disk were not taken into account, which will affect the exact temperature in that region. This does not change the location of the snowlines since they are defined by dust temperatures $T_{\text {dust }} \leq 160 \mathrm{~K}$.

The snowlines of protoplanetary disks without an envelope were obtained and compared with Min et al. (2011) to verify our approach (see Fig. A.1). Using this formulation, the differences in predicted water snowlines are typically within $2 \mathrm{AU}$ at low accretion rates and lower at high accretion rates.

\subsection{Molecular abundances}

The aim of this paper is to calculate the 2D snowlines or snowsurfaces for $\mathrm{CO}, \mathrm{CO}_{2}$, and $\mathrm{H}_{2} \mathrm{O}$ in embedded disks. The region in which these volatiles freeze out onto grains depends on the temperature and density structure (e.g., Meijerink et al. 2009). At steady state, the rate at which the molecule is adsorbed on the grain is balanced by the thermal desorption rate. We adopted a dust number density $n_{\text {dust }}=10^{-12} n_{\mathrm{H}}$ (Visser et al. 2009) with $a_{\text {dust }}=0.1 \mu \mathrm{m}$ as the effective grain size. The number density of solids of species $X$ (see also Fig. B.1) is simply given by

$\frac{n_{\text {ice }}}{n_{\text {gas }}}=\frac{n_{\text {dust }} \pi a_{\text {dust }}^{2}\left(3 k_{\mathrm{B}} T_{\text {gas }} / m_{X}\right)^{1 / 2}}{v_{1} \exp \left(-E_{\mathrm{b}} / T_{\text {dust }}\right) \xi}$,

where $T_{\text {gas }}$ is gas temperature, $T_{\text {dust }}$ is dust temperature, and $m_{\mathrm{X}}$ is the mass of species $X$. The first-order pre-exponential factor, $v_{1}$, was calculated from the binding energy, $E_{\mathrm{b}}$ (Hasegawa et al. 1992; Walsh et al. 2010)

$v_{1}=\sqrt{\frac{2 N_{\mathrm{ss}} E_{\mathrm{b}}}{\pi^{2} m_{\mathrm{X}}}} \mathrm{s}^{-1}$

with the number of binding sites per surface area, $N_{\mathrm{ss}}$, was taken to be $8 \times 10^{14} \mathrm{~cm}^{-2}$ following Visser et al. (2011). A dimensionless factor $\xi\left(n_{\text {ice }}\right)$ was used to switch between zerothorder to first-order desorption when the ice thickness is less than a monolayer. The properties for each molecule are given in Table 2 along with the calculated pre-exponential factor $v_{1}$. These binding energies assume pure ices. The typical timescales for adsorption are $4-6 \times 10^{3} \mathrm{yr}$ at temperature of $50 \mathrm{~K}$ and number densities $\left(n_{\mathrm{H}_{2}}\right)$ of $10^{6} \mathrm{~cm}^{-3}$. This implies that the steadystate assumption is not valid at lower densities that are present in the large-scale envelope $(r>1000 \mathrm{AU})$ where the freezeout timescales become longer than the lifetime of the core (e.g., Jørgensen et al. 2005). On the other hand, we here focus on the 
Table 2. Molecular parameters to calculate $\mathcal{R}_{\text {des }}$ as tabulated in Burke \& Brown (2010).

\begin{tabular}{lccc}
\hline \hline Molecule & $\begin{array}{c}v_{1}^{a} \\
{\left[\mathrm{~s}^{-1}\right]}\end{array}$ & $\begin{array}{c}E_{\mathrm{b}} \\
{[\mathrm{K}]}\end{array}$ & Refs. \\
\hline $\mathrm{CO}$ & $6.4 \times 10^{11}$ & 855 & Bisschop et al. (2006) \\
$\mathrm{CO}_{2}$ & $8.2 \times 10^{11}$ & 2400 & Gálvez et al. (2007) \\
$\mathrm{H}_{2} \mathrm{O}$ & $2.1 \times 10^{12}$ & 5773 & Fraser et al. (2001) \\
\hline
\end{tabular}

Notes. ${ }^{(a)}$ First order desorption pre-exponential factor calculated from the binding energies.

gas phase abundances at small-scale $r \leq 100 \mathrm{AU}$ where the number densities are $n_{\mathrm{H}_{2}}>10^{6} \mathrm{~cm}^{-3}$. Photodesorption can be ignored at such high densities and within the disk, but it may be important at the disk's surface and along the outflow cavity wall. Since the water vapor will also be rapidly photodissociated in these locations, these regions are not major water reservoirs (see Fig. 1). To approximate this region, water was assumed to be absent within a region that is characterized by $A_{\mathrm{V}} \leq 3$ or $N_{\mathrm{H}} \leq 6 \times 10^{21} \mathrm{~cm}^{-2}$. A more detailed gas phase abundance structure through a chemical network will be explored in the future.

\section{Results}

\subsection{Thermal structure of an actively accreting embedded disk}

The locations where various molecules can thermally desorb from the dust grains depend on the temperature structure of the disk. Irradiated disks have a warm upper layer with a cooler midplane. Figure 3 (red dashed lines) shows the midplane (bottom) and vertical temperature at a number of radii (top) for an embedded disk passively irradiated by a $1 L_{\odot}$ central source. Here, we present the results for a $0.1 M_{\odot}$ disk embedded in a $1 M_{\odot}$ envelope whose $R_{\text {cen }}$ is $200 \mathrm{AU}$ (Fig. 3 top). These canonical parameters are highlighted in Table 1.

The dust temperatures of an embedded actively accreting disk are indicated by the blue lines in Fig. 3. The dotted line shows the viscous temperature $\left(T_{\text {visc }}\right)$ as expected at the photosphere (optically thin), while the solid blue lines show the effective dust temperature corrected for the optical depth and passive irradiation. As previously found, the addition of viscous heating can raise the temperatures in the inner few $\mathrm{AU}$ to $>1000 \mathrm{~K}$ (e.g., Calvet et al. 1991; D’Alessio et al. 1997; Davis 2005). The disk temperature is above the water sublimation temperature out to $R \sim 30 \mathrm{AU}$ for an accretion rate of $10^{-5} M_{\odot} \mathrm{yr}^{-1}$. Because of the $R^{-3}$ dependence of $T_{\text {visc }}$ (see Eq. (6)), the viscous heating is dominant in the inner few AU as indicated in Fig. 3 (bottom). Consequently, the passive irradiation due to the central luminosity $\left(L_{\star}\right)$ dominates the temperatures along the disk photosphere and the outflow cavity wall, while the viscous dissipation dominates the heating deep within the disk. These effects can be seen in the 2D dust temperature structure in the inner $\sim 40$ AU shown in Fig. 4 for three different accretion rates. For $\dot{M} \gtrsim 10^{-5} M_{\odot} \mathrm{yr}^{-1}$, panels show the vertical temperature inversions.

The dust temperature structure is also compared with a disk with and without an envelope whose $R_{\text {cen }}$ is $50 \mathrm{AU}$ in Fig. 5. The difference is small in the inner disk and primarily lies at large radii $(R>1 \mathrm{AU})$ where the midplane temperature structure $\left(T_{\text {mid }} \propto \Sigma\right)$ is weakly affected by the adopted envelope model. The temperature structure of the embedded disk model slightly
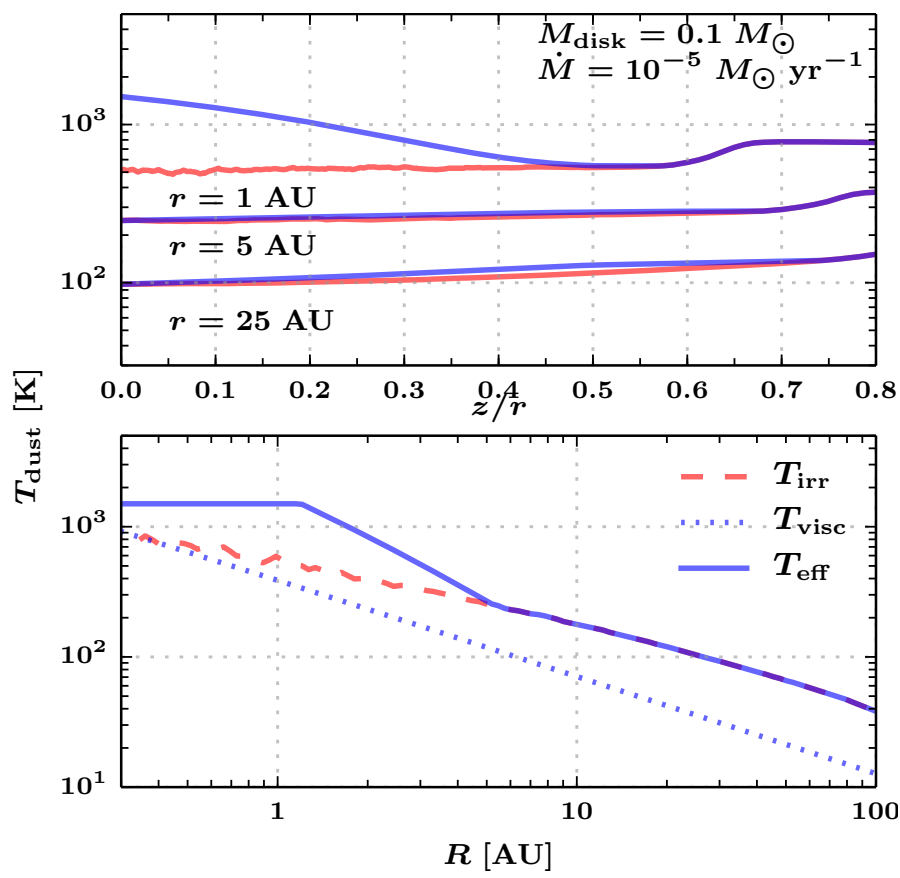

Fig. 3. Midplane radial (bottom) and vertical temperatures (top) at 1, 5, and $25 \mathrm{AU}$ for an embedded $0.1 M_{\odot}$ disk and an accretion rate of $10^{-5} M_{\odot} \mathrm{yr}^{-1}$ irradiated by $L_{\star}=1 L_{\odot}$. The red dashed (bottom) and solid (top) lines indicate the thermal structure of a passively irradiated disk $\left(T_{\text {irr }}\right)$ calculated by the Monte Carlo simulation. The blue solid lines indicate the temperatures including the viscous heating $\left(T_{\text {eff }}=\left(\frac{3}{16} \kappa_{\mathrm{R}} \Sigma_{\text {gas }} T_{\text {visc }}^{4}+T_{\text {irr }}^{4}\right)^{1 / 4}\right)$. The viscous temperature as calculated from Eq. (6) is indicated by the dotted blue lines.

depends on the adopted envelope model. Most importantly, at $R=5$ and $150 \mathrm{AU}$, the embedded disk model with $R_{\text {cen }}=50 \mathrm{AU}$ is warmer than the other two models. This is mainly due to the mass distribution in the inner $100 \mathrm{AU}$. Overall, however, the differences are small (see also D'Alessio et al. 1997) and, for our purposes, it is sufficient to fix the envelope mass to $1 M_{\odot}$ to assess the overall temperature structure of an embedded actively accreting disk. The effects are further discussed in Sect. 3.4. However, the difference at larger radii is sufficient to affect the dominant phase of $\mathrm{CO}_{2}$ and $\mathrm{CO}$.

\subsection{Water snowline}

Using the obtained dust temperatures, the gas and ice number densities were calculated at each cell. To determine the snowline, the total available water mass was computed by adopting a water abundance of $10^{-4}$ with respect to $\mathrm{H}_{2}$. The total available mass was then multiplied by the gas fraction to determine the total water-vapor mass. A minimum gas water abundance of $10^{-9}$ with respect to $\mathrm{H}_{2}$ was used. The snowline is defined as the radius at which $50 \%$ of the total available water has frozen onto the grains $\left(M_{\text {gas }} / M_{\text {ice }}=0.5\right.$; e.g., Min et al. 2011). This typically occurs at $\sim 160 \mathrm{~K}$ in the high-density regions $\left(n_{\mathrm{H}} \sim 10^{14} \mathrm{~cm}^{-3}\right.$, see dashed line in Fig. 4).

Figure 6 presents the midplane snowline radius as a function of accretion rate. In the absence of accretion heating, the water snowline is located at $\sim 3 \mathrm{AU}$. This does not strongly depend on the accretion rate until a value of $\dot{M}>10^{-7} M_{\odot} \mathrm{yr}^{-1}$ is reached. The highest water snowline is located at $55 \mathrm{AU}$ for an accretion rate of $10^{-4} M_{\odot} \mathrm{yr}^{-1}$. Although the effective disk midplane 


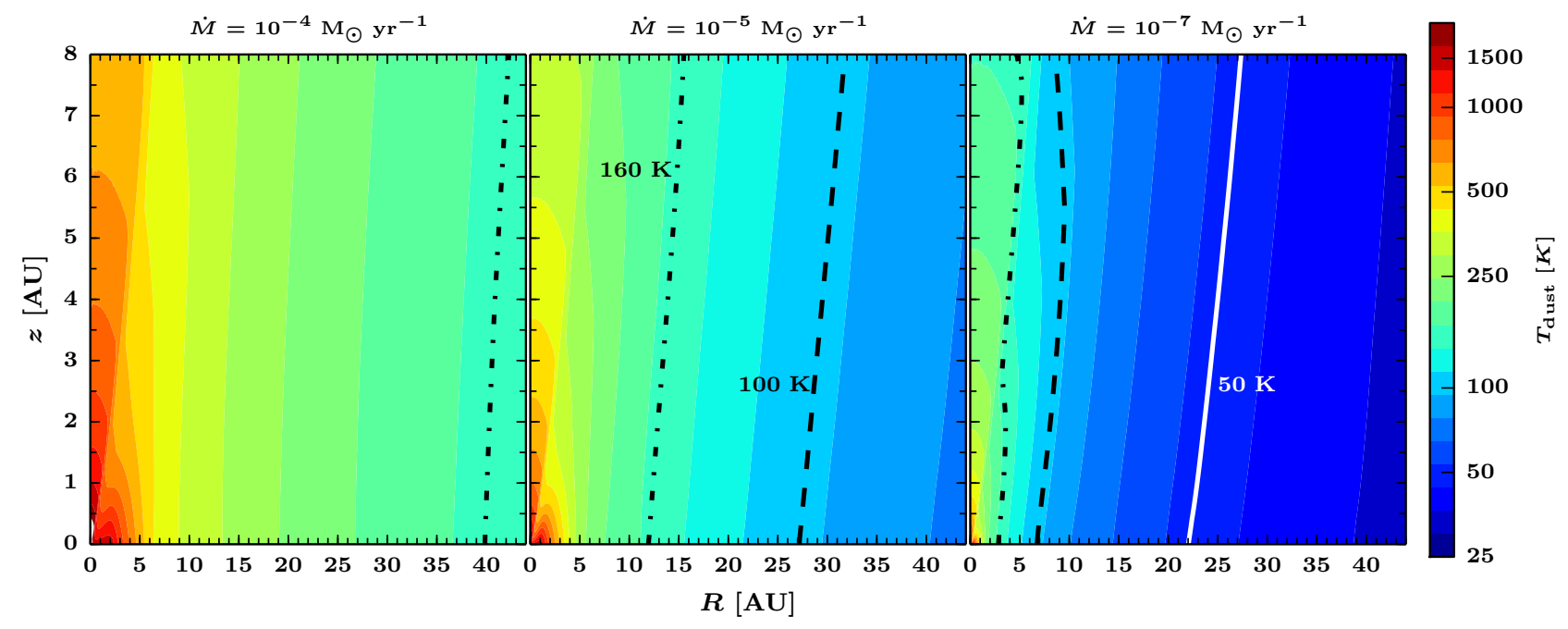

Fig. 4. Dust temperature structure in the inner $44 \mathrm{AU}$ for three different accretion rates for a $0.1 M_{\odot}$ disk embedded in a $1 M_{\odot}$ envelope. A $1 L_{\odot}$ central heating source is adopted for these models. The disk is oriented horizontally, while the outflow cavity is oriented vertically. The three different lines indicate 160 (dashed-dotted), 100 (dashed), and 50 (solid white) K contours, which are important for the water snowlines (see Fig. B.1).

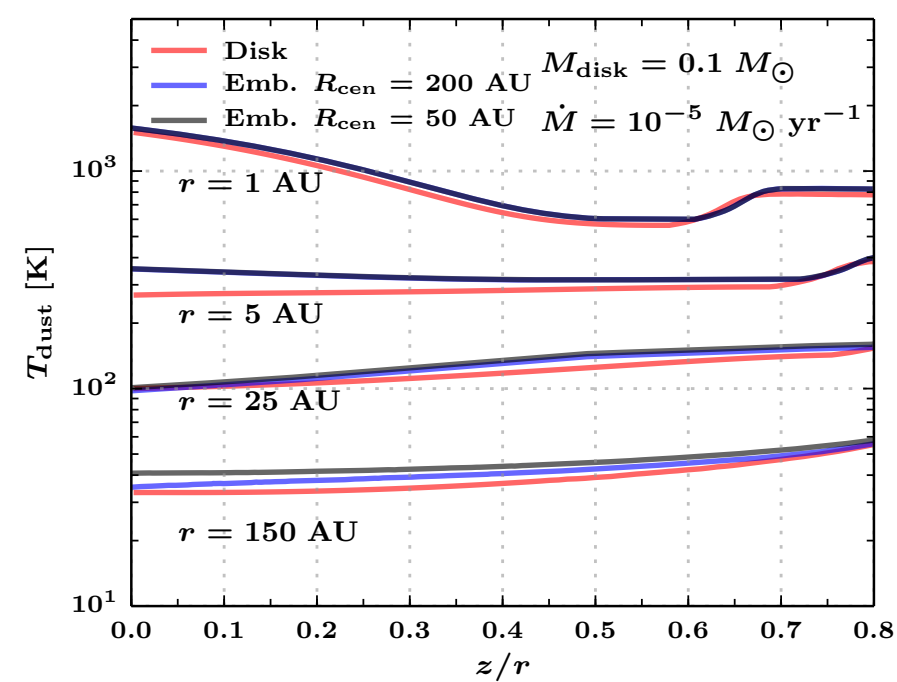

Fig. 5. Comparison of the vertical temperature structure at $1,5,25$, and 150 AU between a protoplanetary disk (red) and embedded disks (blue and black). The difference between the two embedded disk models is the centrifugal radius $R_{\text {cen }}=50 \mathrm{AU}$ (black) versus $200 \mathrm{AU}$ (blue). The envelope mass is $1 M_{\odot}$ for the embedded disk model.

temperature depends on the disk mass, it does not strongly affect the water snowline location. It is located at only slightly smaller radius for a less massive disk $\left(0.05 M_{\odot}\right)$ as indicated in Fig. 6 .

The steep increase of the water snowline at high accretion rates can be understood by comparing the stellar luminosity and the accretion luminosity. For the canonical values that are indicated in Table 1 , the irradiating central luminosity is $1 L_{\odot}$. The accretion luminosity is estimated by integrating Eq. (6) radially over the active disk between 0.1 and $200 \mathrm{AU}$, in this case, and is approximately $L_{\text {acc }} \sim 0.5 \times G M_{\star} \dot{M} / R_{\text {in }}$. Thus, the accretion luminosity is equal to that of the central star for $\dot{M} \sim 3 \times 10^{-6} M_{\odot} \mathrm{yr}^{-1}$. The addition of the accretion onto the star due to the gaseous inner disk provides an additional $\sim 10 L_{\odot}$ luminosity at the given accretion rate. Thus, the accretion luminosity starts to contribute to the heating at $\dot{M} \gtrsim 10^{-7} M_{\odot} \mathrm{yr}^{-1}$ for the adopted parameters (see Fig. 2).

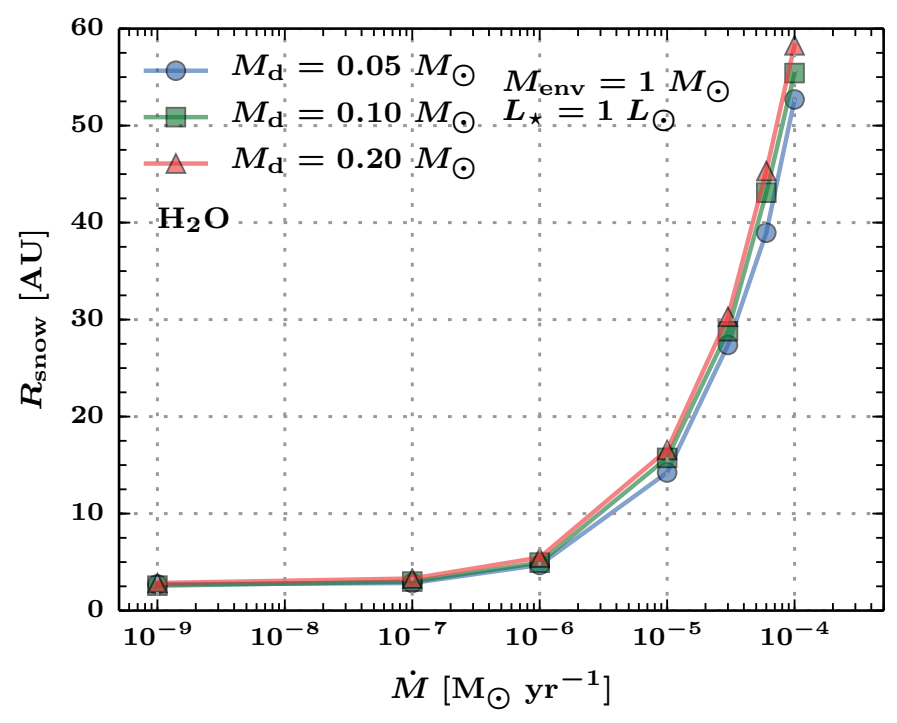

Fig. 6. Midplane water snowlines as function of accretion rates and disk mass for a $200 \mathrm{AU}$ embedded disk with $R_{\text {cen }}=200 \mathrm{AU}$. The different lines indicate the snowline dependence on disk mass at a fixed stellar luminosity and envelope mass.

The observable water emission depends on the water vapor column density. The water vapor column extends farther than the snowline due to the vertical gradient in water vapor abundance. The available water is rapidly frozen out onto dust grains beyond the snowline. To determine whether the location of the snowline is within the disk or not, the hydrostatic disk surface was determined through $H=c_{\mathrm{s}} / \Omega_{\mathrm{K}}$, where $c_{\mathrm{s}}=\sqrt{k_{\mathrm{B}} T_{\text {mid }} / \mu m_{\mathrm{H}}}$ is the sound speed and $\Omega_{\mathrm{K}}$ the Keplerian angular frequency. This is the approximated regime where the gas should be in Keplerian motion. For a $1 L_{\odot}$ central star, most of the water emission arises from the warm inner envelope (sometimes also called the "hot core") above the hydrostatic disk surface as shown by the yellow line in Fig. 7 if a constant water abundance of $10^{-4}$ with respect to $\mathrm{H}_{2}$ is adopted. The blue shaded region in Fig. 7 indicates where water vapor is dominant, some water vapor $(<50 \%$ in mass) is still present within the inner few $\mathrm{AU}$ of the red regions 


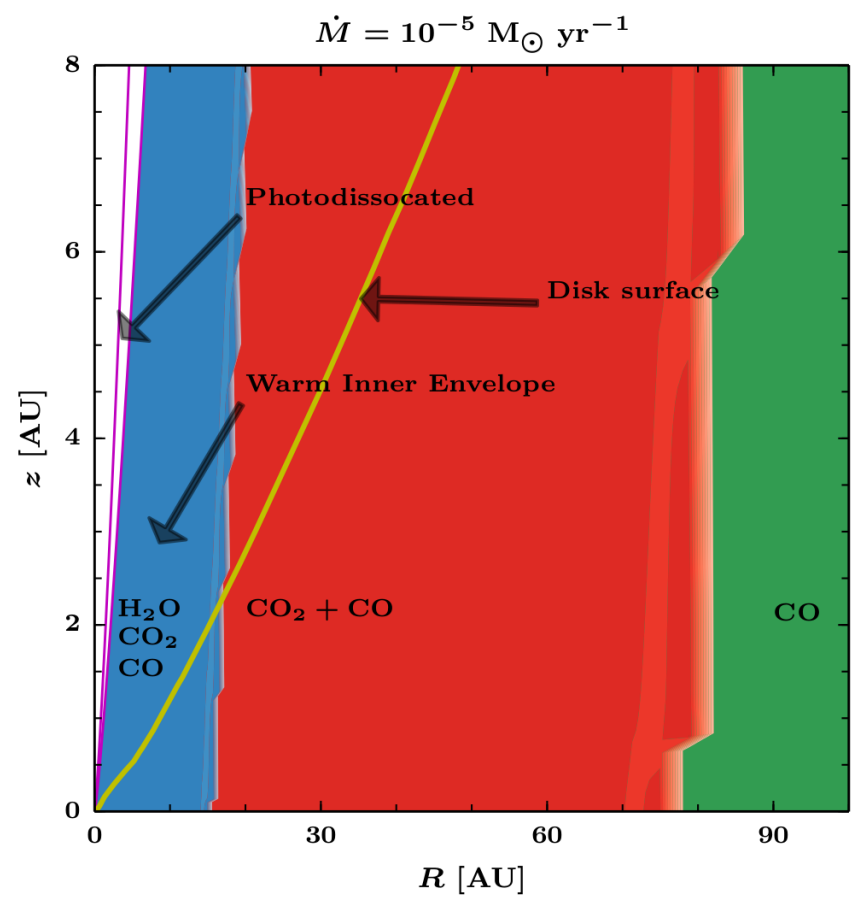

Fig. 7. Locations of gas phase volatiles in the $0.1 M_{\odot}$ disk embedded in a $1 M_{\odot}$ envelope. The different colors indicate the different regimes where various volatiles are found in the gas phase: blue $\left(\mathrm{H}_{2} \mathrm{O}, \mathrm{CO}_{2}\right.$, and $\mathrm{CO}$ ), red (only $\mathrm{CO}_{2}$ and $\mathrm{CO}$ ), and green (only $\mathrm{CO}$ ). The accretion rate is indicated at the top. The arrows are indicating three different regions as indicated in Fig. 1: water photo-dissociation region defined by $A_{\mathrm{V}}=3$, warm inner envelope, and the disk surface (yellow line assuming $T_{\text {gas }}=T_{\text {dust }}$ ).

( $\lesssim 30$ AU). Water vapor is also present along the cavity walls as indicated by the two purple lines in Fig. 7, but these regions have low extinctions $\left(A_{\mathrm{v}}<3\right)$, which allows for the photodissociation of water.

\section{3. $\mathrm{CO}$ and $\mathrm{CO}_{2}$ snowlines}

Pure $\mathrm{CO}_{2}$ and $\mathrm{CO}$ ices thermally desorb over a narrow range of dust temperatures between $40-80 \mathrm{~K}$ and $15-30 \mathrm{~K}$, respectively, depending on the density (see Fig. B.1). As a result of their lower binding energies relative to water, $\mathrm{CO}$ and $\mathrm{CO}_{2}$ are in the gas phase within a large part of the embedded disk. The $\mathrm{CO}_{2}$ snowline is between $20-250 \mathrm{AU}$ for $10^{-9}-10^{-4} M_{\odot} \mathrm{yr}^{-1}$ accretion rates (see Fig. 8). Thus, the entire disk including the midplane lacks $\mathrm{CO}_{2}$ ice for highly acccreting embedded disks $\left(\dot{M} \sim 10^{-4} M_{\odot} \mathrm{yr}^{-1}\right)$. The steep rise of the $\mathrm{CO}_{2}$ snowline at high accretion rates is similar to that of water as shown in Fig. 6.

Since the adopted binding energy of $\mathrm{CO}$ to the dust grain is the least with $855 \mathrm{~K}, \mathrm{CO}$ largely remains in the gas phase within the disk for $L_{\star} \geq 1 L_{\odot}$ independently of the accretion rate (see Fig. 8 right). At low accretion rates, the snowline is located at $\sim 150 \mathrm{AU}$ at the midplane, indicating the presence of $\mathrm{CO}$ ice between 150 to $200 \mathrm{AU}$ within the disk. However, the bulk of $\mathrm{CO}$ within the disk remains in the gas phase as shown in Fig. 7. A smaller disk during the embedded phase would lead to stronger envelope irradiation (e.g., D’Alessio et al. 1997), and, consequently, $\mathrm{CO}$ is not frozen out within the disk. A sufficiently large and massive disk $\left(M_{\text {disk }}>0.2 M_{\odot}\right.$ irradiated by a $1 L_{\odot}$ star $)$ could contain a higher percentage of $\mathrm{CO}$ ice at large radii. This is simply due to the increase of optical depth and, thus, lower dust temperatures at large radii.

\subsection{Dependence on stellar, disk, and envelope properties}

Previous sections presented the vapor content for a $0.1 M_{\odot}$ embedded disk surrounded by a $1 M_{\odot}$ envelope with $R_{\text {cen }}=200 \mathrm{AU}$ that is being irradiated by a $1 L_{\odot}$ central star $\left(T_{\star}=4000 \mathrm{~K}\right.$, $R_{\star}=2.1 R_{\odot}$ ) as highlighted in Table 1. As noted in Sect. 2.2, the effective midplane temperature $\left(T_{\text {eff }}\right)$ depends on the disk mass, but an increasing disk mass only leads to a small change in the water snowline as shown in Fig. 6. Figure 7 shows that the water vapor can be either within the disk or within the warm inner envelope. This section explores how the vapor content depends on disk radius and central luminosity for fixed envelope and disk $\left(0.1 M_{\odot}\right)$ masses.

Figure 9 presents the locations of water snowline as a function of accretion rate, luminosity, and disk radius. For a fixed disk structure, a factor of 5 increase in $L_{\star}$ shifts the midplane water snowline by a factor of $\sim 2$ from $\sim 4 \mathrm{AU}\left(1 L_{\odot}\right)$ to $\sim 10 \mathrm{AU}$ $\left(10 L_{\odot}\right)$ at low accretion rates $\left(\dot{M}<10^{-7} M_{\odot} \mathrm{yr}^{-1}\right)$. A factor of 15 increase in $L_{\star}$ yields a factor of $\sim 4$ increase in the location at the water snowline. The stellar luminosity $L_{\star}$ is defined by the stellar radius, while the accretion dependent total luminosity $L_{\text {tot }}$ that is irradiating the system is shown in Fig. 2. For high accretion rates $\left(\dot{M} \gtrsim 10^{-5} M_{\odot} \mathrm{yr}^{-1}\right)$, the water snowline decreases as $L_{\star}$ increases. This can be explained as due to the fact that $L_{\text {tot }}$ decreases as $L_{\star}$ increases at high accretions because $R_{\star}$ increases, which in turn decreases the luminosity that is provided by the inner gaseous disk. Thus, if the stellar accretion rates are low $\left(L_{\star}>L_{\text {acc }}\right)$, the stellar luminosity is the parameter to be varied to increase the water snowline. However, the water snowline can be up to 50-60 AU if the stellar luminosity is low (small stellar radius) and the stellar accretion rate is high.

The $\mathrm{CO}_{2}$ midplane snowline shows a similar behavior as the water snowline (Fig. B.2) as a function of stellar luminosity and accretion rates. It is in the gas phase at $R>40 \mathrm{AU}$ for $L_{\star} \geq$ $5 L_{\odot}$, which is an increase of a factor of 2 in terms of snowline location with respect to the canonical value of $L_{\star}=1 L_{\odot}$. As $\mathrm{CO}$ is already largely in the gas phase for the canonical values, a small increase of the stellar luminosity results in the lack of a CO snowline within the disk (Fig. B.3).

Another parameter that affects the total energy input into the embedded system is the disk radius. The total accretion luminosity that is being added depends on the disk radius, which is the region where the disk accretion energy is being integrated. A decreasing disk radius leads to a decreasing accretion luminosity. This only affects the midplane water snowline in the case of $L_{\star}=1 L_{\odot}$ (see Fig. 9). In this case, the difference is seen only for high stellar accretion rates where the accretion luminosity $\left(L_{\text {disk }}+L_{\text {in }}\right)$ starts to be the dominant heating source. The midplane water snowline decreases from $\sim 55 \mathrm{AU}$ to $\sim 38 \mathrm{AU}$ as the disk radius decreases from $200 \mathrm{AU}$ to 50 AU. Thus, an increasing stellar luminosity $\left(L_{\star}\right)$ has a greater effect on the midplane water snowline. This leads to an increase of water vapor in the warm inner envelope (see Fig. 7) for low stellar accretion rates $\left(\dot{M}<10^{-5} M_{\odot} \mathrm{yr}^{-1}\right)$. However, if $L_{\star}>1 L_{\odot}$, the disk radius does not affect the location of the midplane water snowline even for high accretion rates $\left(\dot{M}>10^{-5} M_{\odot} \mathrm{yr}^{-1}\right)$ since the total luminosity is dominated by the stellar luminosity. The $\mathrm{CO}_{2}$ snowline, on the other hand, shows a stronger dependence on the disk radius than that of water. For the high stellar luminosity case $L_{\star}=15 L_{\odot}$, the $\mathrm{CO}_{2}$ snowline increases from $40 \mathrm{AU}$ to $80 \mathrm{AU}$ as the disk radius $\left(R_{\text {disk }}\right)$ increases from 50 AU to $200 \mathrm{AU}$. This can be explained by the density distribution in the inner region. For a small disk, the dust temperature is low enough at large radii such that $\mathrm{CO}_{2}$ can exist in the solid phase. However, as 

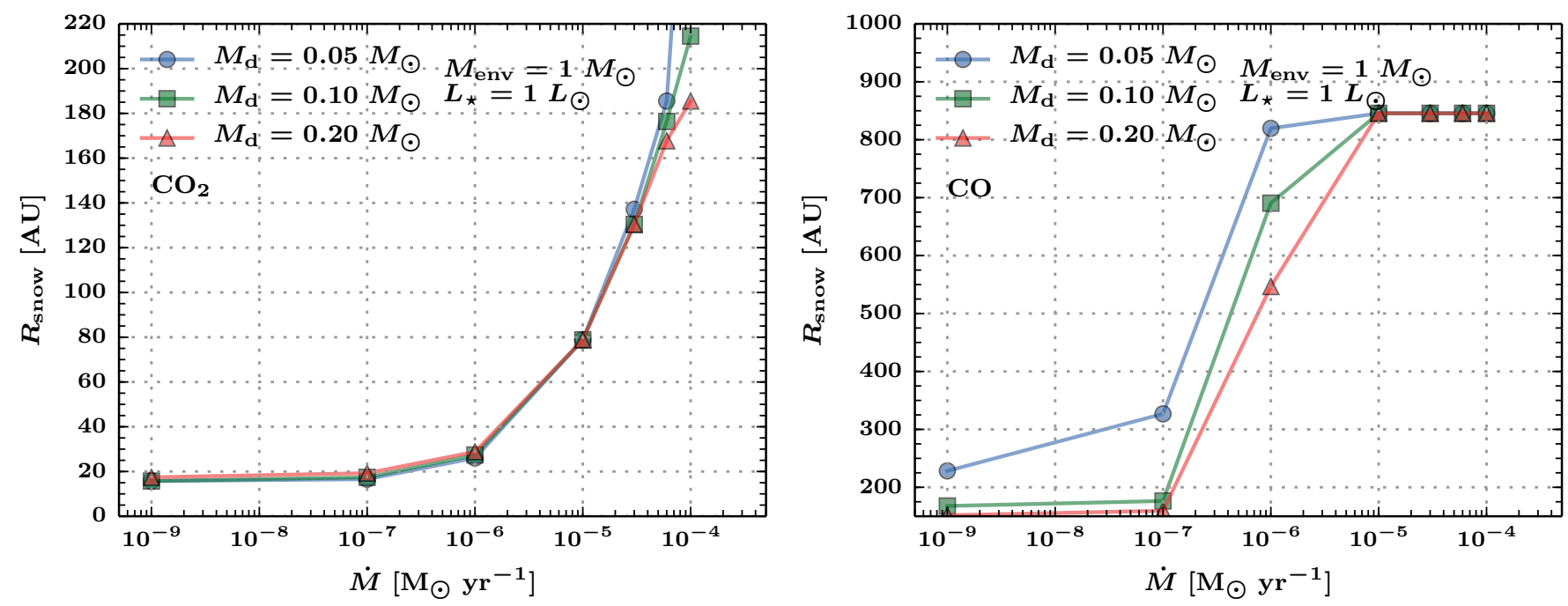

Fig. 8. Left: midplane $\mathrm{CO}_{2}$ snowlines as a function of accretion rates and disk mass for a $200 \mathrm{AU}$ disk. Right: midplane $\mathrm{CO}$ snowlines as a function of accretion rates for the same disk. The different colors indicate the snowline dependence on disk mass at a fixed stellar luminosity $\left(1 L_{\odot}\right)$ and envelope mass $\left(1 M_{\odot}\right)$.

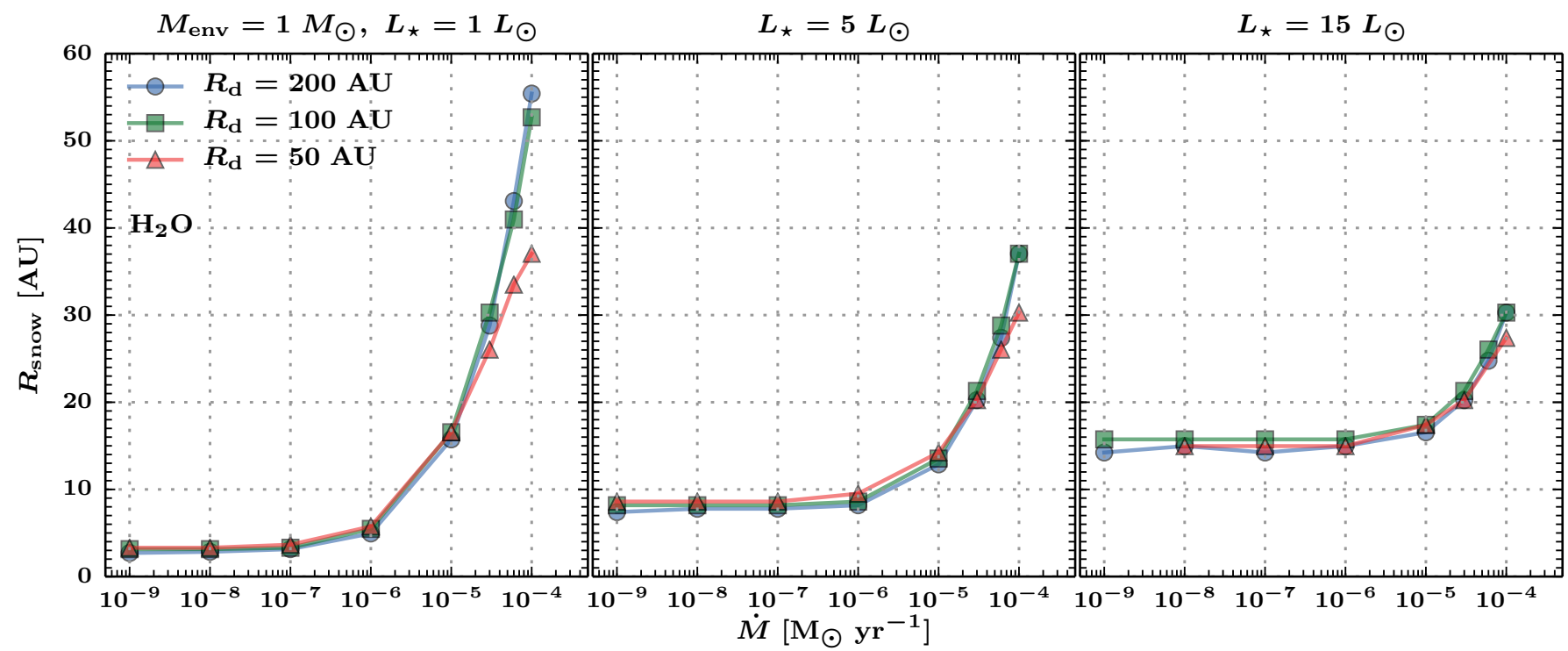

Fig. 9. Midplane water snowline as a function of stellar luminosity, accretion rate, and disk radius. The envelope mass is fixed at $1 M_{\odot}$ with a disk mass of $0.1 M_{\odot}$ and $R_{\text {cen }}=200 \mathrm{AU}$. The different panels show the water snowline as a function of stellar luminosity: $1 L_{\odot}($ left $), 5 L_{\odot}(c e n t e r)$, and $15 L_{\odot}($ right). The different colors indicate the disk radius: $200 \mathrm{AU}$ (blue), $100 \mathrm{AU}$ (green), and $50 \mathrm{AU}$ (red).

the size of the disk increases, the overall density at similar radii decreases, which results in lower optical depth and, as consequence, higher dust temperatures at large radii. The disk radius parameter seems to be more important for the $\mathrm{CO}_{2}$ snowline than for the water snowline.

The main parameter for the envelope in our setup is the centrifugal radius. For the canonical value of $R_{\text {cen }}=200 \mathrm{AU}$, the mass of the disk can be distributed between 50,100, or $200 \mathrm{AU}$. The disk radius $R_{\text {disk }}$ is where the disk ends, and it can be smaller than $R_{\text {cen. }}$. Here, the effects of a flattened envelope as defined by the centrifugal radius $R_{\text {cen }}$ are presented. A smaller centrifugal radius results in a hotter inner disk (see Fig. 5). However, the affected regions correspond to the regions within the disk where dust temperatures are already $>100 \mathrm{~K}$. Thus, we find that there is no change in the water snowline for a more flattened envelope structure $\left(R_{\text {cen }}=50 \mathrm{AU}\right.$ instead of $\left.200 \mathrm{AU}\right)$. More volatile species such as $\mathrm{CO}_{2}$ and $\mathrm{CO}$ are affected by the flattened envelope structure, however. The $\mathrm{CO}_{2}$ snowline is confined within the inner $40 \mathrm{AU}$ for $\dot{M} \leq 10^{-5} M_{\odot} \mathrm{yr}^{-1}$. At higher accretion rates, the combination of a warm disk and low envelope densities at $R>50 \mathrm{AU}$ for the $R_{\text {cen }}=50 \mathrm{AU}$ model results in a warm envelope where $\mathrm{CO}_{2}$ is largely in the gas phase up to 400-500 AU along the midplane, while it is $200 \mathrm{AU}$ in the canonical model. The snowline of CO is already at $400 \mathrm{AU}$ for an $R_{\text {cen }}=50 \mathrm{AU}$ envelope model at low accretion rates instead of $\sim 200-300$ AU in the case of $R_{\text {cen }}=200$ AU envelope. Thus, we find that the inner envelope and disk physical structure to be important in determining the location of snowlines of various volatiles. These parameters are largely unknown as yet for deeply embedded YSOs. 


\section{Discussion}

\subsection{Comparison with accreting protoplanetary disk models}

In the absence of an envelope, similar stellar parameters and an accretion rate of $10^{-8} M_{\odot} \mathrm{yr}^{-1}$ lead to the midplane water snowline located at $\sim 1$ AU (e.g., Sasselov \& Lecar 2000). Lecar et al. (2006) suggested that the snowline can move out by increasing the accretion rate, disk mass, and the dust opacities. The differences between the derived snowlines in the literature are unlikely to be due to the differences in radiative transfer treatment, which has been compared in Min et al. (2011). As an example, Garaud \& Lin (2007) derived similar water snowlines to that of Min et al. (2011; 1 AU vs. 2 AU) at similar accretion rates using different methods in deriving the dust temperatures and different opacities. The latter model takes into account the detailed 2D vertical structure of the disk and dust sublimation front. As Min et al. (2011) showed, most of the differences are due to the adopted dust opacities. Our value is $\sim 1$ AU higher than the values tabulated in Min et al. (2011) for similar parameters. Furthermore, most of the previous studies adopted the minimum mass solar nebula (MMSN) model, where $\Sigma \propto R^{-1.5}$ instead of the $R^{-1} 2 \mathrm{D}$ parametric disk structure used in this paper (see, e.g., Andrews et al. 2009). Previous snowlines or snow surfaces studies did not take the flattened envelope into account.

Previous studies have found that the water snowlines in the more evolved disks decrease with decreasing accretion rates (e.g., Davis 2005; Garaud \& Lin 2007; Min et al. 2011). This has been reproduced in Fig. A.1. However, if the disk structure is related to its stellar accretion rate such as adopted in Garaud $\&$ Lin (2007), the water snowline increases to a constant value at $\sim 2 \mathrm{AU}$ for very low accretion rates $\left(\dot{M} \lesssim 10^{-10} M_{\odot} \mathrm{yr}^{-1}\right)$. This is due to the disk becoming more optically thin, which in turn allows for stellar photons to penetrate deeper radially into the disk. In this paper, the disk structure was fixed while the stellar accretion rate was varied. However, the envelope provides a blanket where the disk can stay relatively warm. Thus, even though the disk structure is fixed, the snowline stays at a fixed radius for decreasing stellar accretion rates.

Taking the envelope into account, for the same accretion rate of $10^{-8} M_{\odot} \mathrm{yr}^{-1}$, our water snowline is at $\sim 3 \mathrm{AU}$ for embedded disks compared to 1-2 AU without an envelope. The presence of the envelope does not strongly affect the midplane temperature at regions close $100 \mathrm{~K}$, as shown in Fig. 5. Thus, it does not significantly modify the water snowline. However, it does affect the $\mathrm{CO}_{2}$ and $\mathrm{CO}$ snowlines, since the $40 \mathrm{~K}$ region shifts inward under the presence of an envelope depending on the centrifugal radius.

\subsection{Caveats}

The effect of convection in the vertical direction is not included in this study. It is typically found to be important in the case of high accretion rates $\left(\dot{M}>10^{-6} M_{\odot} \mathrm{yr}^{-1}\right.$, D'Alessio et al. 1998; Min et al. 2011). Convection is found to cool the midplane temperatures at $T_{\text {dust }}>500 \mathrm{~K}$. Thus, this should not affect the water $(100-160 \mathrm{~K}), \mathrm{CO}_{2}(\sim 50 \mathrm{~K})$ and $\mathrm{CO}(\sim 20 \mathrm{~K})$ snowlines as their sublimation temperatures are much lower than $500 \mathrm{~K}$.

Under the assumption of the steady-state accretion disk model, the typical values of $\alpha$ are found to be $>1$ for an accretion rate of $\dot{M}=10^{-4} M_{\odot} \mathrm{yr}^{-1}$. This is significantly higher than that expected from magnetorotational instability (MRI) driven accretion in an MHD disk ( $\alpha=0.01$, Balbus \& Hawley 1998). King et al. (2007) indicated that $\alpha$ could be $\sim 0.4$ for thin disks around compact systems. These high $\alpha$ values in our models are obtained because we fixed the density distribution at a high accretion rate $\left(\dot{M}>10^{-5} M_{\odot} \mathrm{yr}^{-1}\right)$. In reality, such a disk is unphysical. However, this is only encountered for the highest accretion rate of $10^{-4} M_{\odot} \mathrm{yr}^{-1}$ that occurs for a very short time. In principle, this can be avoided by iterating the disk structure to ensure that the hydrostatic conditions are satisfied. However, this paper explores the effect of the adopted accretion rates and disk masses for a given fixed set of parameters. Self-consistent models including gas opacities and multiple dust species should be explored in the future.

\subsection{Comparison with observations}

The extent of the observed spatially resolved water emission toward low-mass embedded YSOs is between 25-90 AU (Jørgensen \& van Dishoeck 2010; Persson et al. 2012, 2014). The water snowline is confined to within the inner $40 \mathrm{AU}$ for the cases of the envelope models whose centrifugal radius is $50 \mathrm{AU}$. In such models, we set the disk to not extend beyond $50 \mathrm{AU}$. The water snowline was only increased when the centrifugal radius of the envelope was larger than $50 \mathrm{AU}$, in which case the water snowline can extend up to $55 \mathrm{AU}$ if the stellar luminosity is low $\left(L_{\star}=1 L_{\odot}\right)$. Thus, our results indicate that most of the water emission at $R<50 \mathrm{AU}$ would originate from the combined disk and warm inner envelope (see Fig. 7).

Through the combined modeling of the continuum spectral energy distribution and submm continuum images, the envelope masses toward the observed embedded sources (NGC 1333 IRAS 2A, IRAS 4A, and IRAS 4B) are found to be between 3 $5 M_{\odot}$ (Kristensen et al. 2012), somewhat higher than our canonical value. On the other hand, all three sources are binaries (e.g., Jørgensen et al. 2004, 2007), therefore it is likely that the effective envelope mass that affects the disk physical structure is lower than the above values. Therefore, the envelope masses were kept fixed at $1 M_{\odot}$.

The amount of water vapor in the embedded disk models can be compared to the observed optically thin millimeter water emission toward deeply embedded YSOs. Thermalized $\mathrm{H}_{2}^{18} \mathrm{O}$ emission at $203.4 \mathrm{GHz}\left(3_{1,3}-2_{2,0}\right)$ is calculated using the following equation

$$
\begin{aligned}
I_{v} & \approx B_{v}\left(T_{\mathrm{ex}}\right)\left(1-e^{-\tau}\right), \\
\tau & =\frac{\bar{N}}{Q\left(T_{\mathrm{ex}}\right) \Delta V} \frac{A_{\mathrm{ul}} c^{3}}{8 \pi v^{3}} e^{h v / k_{\mathrm{B}} T_{\mathrm{ex}}},
\end{aligned}
$$

where $A_{\mathrm{ul}}$ is the Einstein $A$ coefficient of the $203 \mathrm{GHz}$ transition is $4.5 \times 10^{-7} \mathrm{~s}^{-1}, T_{\text {ex }}$ is taken to be $150 \mathrm{~K}, E_{\mathrm{u}}=204 \mathrm{~K}$ is the upper energy level, and $Q$ is the temperature-dependent partition function adopted form the HITRAN database (Rothman et al. 2009). The beam-averaged column density $(\bar{N})$ is taken from within $50 \mathrm{AU}$ radius with ${ }^{16} \mathrm{O}:{ }^{18} \mathrm{O}$ isotopic ratio of 540 (Wilson $\&$ Rood 1994). The line profile is assumed to be Gaussian with a FWHM $(\Delta v)=1-4 \mathrm{~km} \mathrm{~s}^{-1}$ as observed toward the three embedded sources reported by Persson et al. (2014).

Figure 10 (left) presents the mean $\mathrm{H}_{2}^{18} \mathrm{O}$ gas column density of the simulated embedded disks as a function of accretion rate for different disk masses and stellar luminosities. The mean column density rises sharply for $\dot{M}>10^{-7} M_{\odot} \mathrm{yr}^{-1}$ as expected for a $1 L_{\odot}$ stellar luminosity, while the sharp rise occurs at $\dot{M}>10^{-6} M_{\odot}$ for a $5 L_{\odot}$ irradiating source. The typical line optical depth at the line center $\left(v=0 \mathrm{~km} \mathrm{~s}^{-1}\right)$ can be $\tau_{v=0} \geq 1$. The typical average column densities of water between $50 \mathrm{AU}$ to $1000 \mathrm{AU}$ are $>4$ orders of magnitude lower than the values 

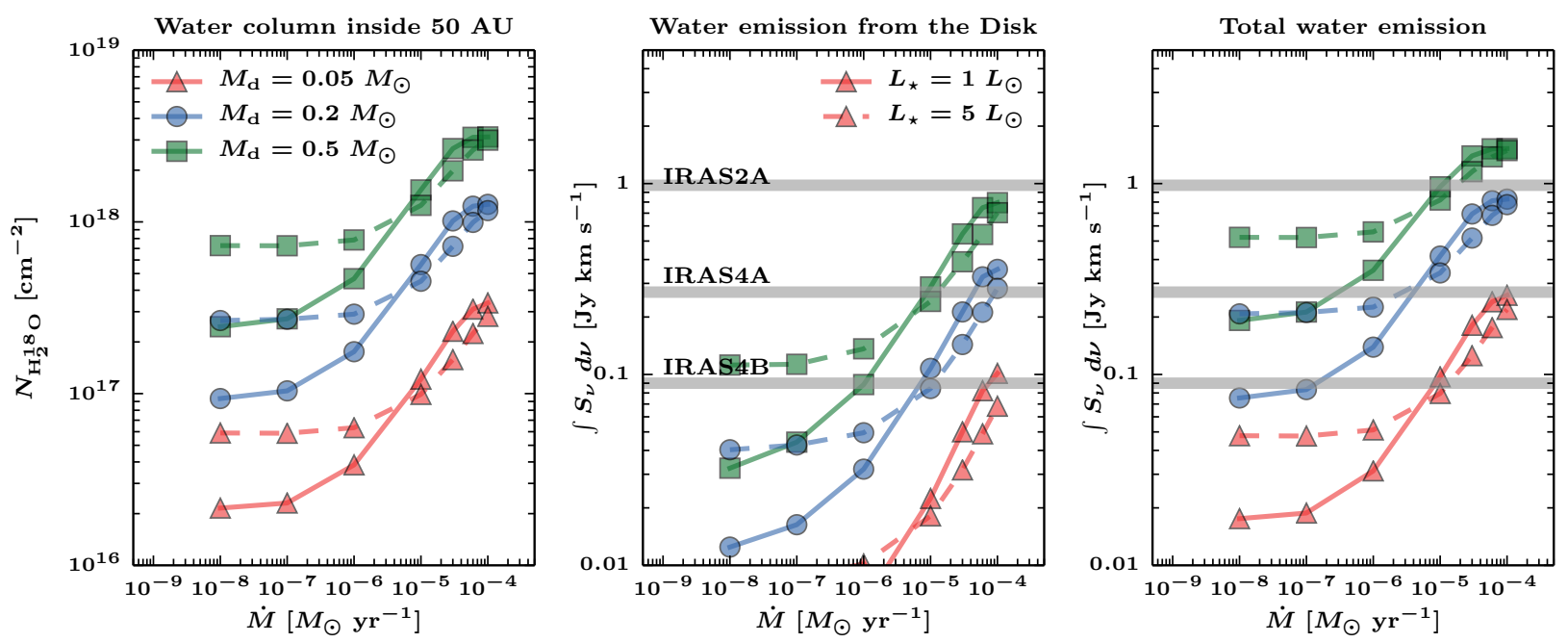

Fig. 10. Left: beam-averaged water $\left(\mathrm{H}_{2}^{18} \mathrm{O}\right)$ column densities within $50 \mathrm{AU}$ radius as a function of accretion rates. The different colors indicate the disk mass dependence for $R_{\text {disk }}=200$ AU models: $M_{\mathrm{d}}=0.05 M_{\odot}$ (red triangles), 0.2 (blue circles), and 0.5 (green squares). The different lines show the luminosity dependence: $L_{\star}=1 L_{\odot}$ (solid) and $5 L_{\odot}$ (dashed). Center: integrated line flux densities arising from the disk as defined by the yellow line in Fig. 7. The observed integrated $\mathrm{H}_{2}^{18} \mathrm{O}$ line is indicated by the gray lines for the three different embedded YSOs in Persson et al. (2014). Right: integrated line flux densities accounting for the entire water vapor mass assuming a Gaussian line width of $1 \mathrm{~km} \mathrm{~s}^{-1}$, which is appropriate for NGC 1333-IRAS 4B.

within $50 \mathrm{AU}$ as presented in Fig. 10. Thus, the water present within $50 \mathrm{AU}$ dominates the total water mass for the adopted models.

The expected integrated $\mathrm{H}_{2}^{18} \mathrm{O} 3_{1,3}-2_{2,0}(203.4 \mathrm{GHz})$ line flux densities for the embedded disk models are also shown in Fig. 10 (right). The integrated line flux densities $\left(\int S_{\nu} \mathrm{d} v\right)$ were calculated with a Gaussian line profile with $\Delta v=1 \mathrm{~km} \mathrm{~s}^{-1}$ (1.06 $S_{v=0} \times \Delta v$, where $S_{v=0}$ is the peak flux density at line center) as observed toward NGC 1333-IRAS 4B (Persson et al. 2014). The observed line widths toward IRAS $2 A$ and IRAS 4A are 4 and $3 \mathrm{~km} \mathrm{~s}^{-1}$, respectively. Increasing $\Delta v$ slightly increases the model integrated line flux densities because the line is slightly optically thick.

The predicted integrated line flux densities from actively accreting embedded disk models are consistent with that observed toward NGC 1333 IRAS 4B if $M_{\mathrm{d}} \leq 0.2 M_{\odot}$ with $\dot{M} \gtrsim$ $6 \times 10^{-7} M_{\odot} \mathrm{yr}^{-1}$ for $L_{\star}=1 L_{\odot}$. Jørgensen et al. (2009) estimated from the continuum that the disk mass is $\sim 0.2 M_{\odot}$, which is consistent with our results. Decreasing the disk mass implies that a higher accretion rate is required to reproduce the observed water emission. A higher accretion rate $\left(\sim 1 \times 10^{-5} M_{\odot} \mathrm{yr}^{-1}\right)$ with lower disk mass is also consistent with the observed extent of water emission at $\sim 20 \mathrm{AU}$. A similar conclusion is reached for the case of IRAS 4A, whose disk is estimated to be $\sim 0.5 M_{\odot}$ (Jørgensen et al. 2009). Since the bolometric luminosities toward NGC 1333 IRAS 4A and IRAS 4B are a factor of 4-9 higher than the adopted central luminosity $\left(L_{\star}\right)$, a model with a factor of 2 lower mass disk can also reproduce the observed flux density with accretion rates $\sim 10^{-5} M_{\odot} \mathrm{yr}^{-1}$. The total luminosity generated by such embedded systems would be similar to the observed bolometric luminosities. With such a lower mass disk, the warm inner envelope is the dominant source of emission instead of the Keplerian disk. This implies that it would be difficult to observe the Keplerian motion of the disk from $\mathrm{H}_{2}^{18} \mathrm{O}$ lines.

IRAS 2A shows stronger integrated line emission $\left(\sim 1 \mathrm{Jy} \mathrm{km} \mathrm{s}^{-1}\right)$ relative to the other two sources and extending up to $90 \mathrm{AU}$ radius. As pointed out above, a disk cannot be responsible for any warm water emission at $R>50 \mathrm{AU}$. Furthermore, Brinch et al. (2009) estimated that the disk is at most $\sim 0.05 M_{\odot}$, which lowers the expected disk contribution to the observed water emission (see also Jørgensen et al. 2009 and Maret et al. 2014). There are two possible scenarios that can explain such a high water emission: a more luminous star $\left(L_{\star}>10 L_{\odot}\right)$ or a high stellar accretion rate $\left(\sim 10^{-5} M_{\odot} \mathrm{yr}^{-1}\right)$. Both solutions result in total luminosity $L_{\text {tot }}$ comparable to the observed bolometric luminosity of IRAS $2 \mathrm{~A}\left(L_{\mathrm{bol}}=35.7 L_{\odot}\right.$, Karska et al. 2013). In either case, most of the water emission arises from the warm inner envelope with negligible disk contribution.

The best cases for an actively accreting embedded disk scenario are IRAS 4A and IRAS 4B. More detailed modeling of their physical structure at $<100$ AU radius is required to further constrain the relation between infall (envelope to disk) and stellar accretion rate (disk to star). Mottram et al. (2013) suggests that the infall rate from the large-scale envelope toward IRAS 4A at $1000 \mathrm{AU}$ to be $\sim 10^{-4} M_{\odot} \mathrm{yr}^{-1}$. For disk masses between $0.2-0.5 M_{\odot}$, the disk must also process the material at similar rate (within a factor of 10), which is consistent with the models presented here.

Observational perspectives. Future spatially and spectrally resolved ALMA observations of optically thin water $\left(\mathrm{H}_{2}^{18} \mathrm{O}\right)$ and deuterated water (HDO) lines may be able to differentiate these models. The spatially resolved data can provide the extent of the water emission. The comparison between the source's bolometric luminosity and the extent of the water emission can provide limits on the stellar accretion rate. A low bolometric luminosity source cannot have a water emission originating from the disk farther than a few AU. Furthermore, the velocity information within the inner $50 \mathrm{AU}$ radius can help in distinguishing between the disk and the infalling envelope (see, e.g., Harsono et al. 2015).

\subsection{Connecting to the young solar nebula}

It is thought that the disk out of which the solar system formed (the solar nebula) was initially hot enough to vaporize all material inherited from the collapsing cloud into atoms, then 
turn them into solids according to a condensation sequence as the nebula cools (Lewis 1974; Grossman \& Larimer 1974). Evidence for energetic processing and subsequent condensation comes from meteoritic data collected in the inner solar system (see Kerridge \& Matthews 1988; Scott 2007; Apai \& Lauretta 2010, for reviews). After the refractory phases have formed, more volatile species such as ices can also condense at larger distances from the young Sun, with ice composition depending on the temperature and pressure as well as the elemental abundance ratios (e.g., Lunine et al. 1991; Owen \& Bar-Nun 1993; Mousis et al. 2009; Pontoppidan et al. 2014). Using cooling curves appropriate for the solar nebula, models show that various ices including $\mathrm{H}_{2} \mathrm{O}, \mathrm{CH}_{3} \mathrm{OH}, \mathrm{NH}_{3}$, and $\mathrm{CO}_{2}$ ice form by condensation out of warm gas out to at least 20 AU (Mousis et al. 2012; Marboeuf et al. 2014). This condensation process is important to explain the composition of volatiles in the atmospheres of solarsystem giant planets and comets.

Where and when does this heating and condensation in the disk actually occur? The above processes are usually discussed in the context of protoplanetary disks where the envelope has dissipated and where the water snowline is eventually at a few AU once the disk has cooled. However, neither observations nor models of disks around solar-mass T Tauri stars show any evidence that disks are as warm as required above to have condensation occurring out to $20 \mathrm{AU}$ (Beckwith et al. 1990; D'Alessio et al. 1998; Dullemond et al. 2007). Furthermore, observations suggest that significant grain growth has occurred (Testi et al. 2014) and disk inhomogeneities due to protoplanets are present in such evolved disks (e.g., Quanz et al. 2013). This further violates the assumption that the physical structure of an evolved disk can be used to study the young solar nebula. The results from this paper indicate that instead such a hot disk may be more common in the early stages of disk formation during the embedded phase $\left(M_{\mathrm{env}}>M_{\mathrm{disk}}\right)$. The disk is then expected to have high accretion rates providing the necessary additional heating. Furthermore, the high accretion rates necessary to push the midplane snowlines to $20 \mathrm{AU}$ and beyond tend to occur for only a short time (e.g., Vorobyov 2009). Most volatiles including $\mathrm{H}_{2} \mathrm{O}$, $\mathrm{CH}_{3} \mathrm{OH}, \mathrm{CO}_{2}$, and $\mathrm{CO}$ presented here will then be in the gasphase within this 20-30 AU radius through sublimation of ices. Shortly after, the volatiles recondense as the accretion rate decreases, with the process controlled by the freeze-out timescale. The inclusion of the gas motions (radially and vertically) and kinetics does not allow for instantaneous re-condensation, thus some gas-phase volatiles are expected to remain near the snowline (Lewis \& Prinn 1980; Ciesla \& Cuzzi 2006).

A related question is whether the volatiles that are incorporated into planetesimals and eventually planets and icy bodies in the critical 5-30 AU zone are then inherited or reset during the disk formation process. The material is defined to be inherited if the simple and complex ices that have been built up in the envelope survive the voyage to the planetary and cometary forming zones. Evolutionary models by Visser et al. (2009) and Visser et al. (2011) suggest that strongly bound ices such as $\mathrm{H}_{2} \mathrm{O}$ are largely pristine interstellar ices in the outer disk, whereas more volatile species can have sublimated, recondensed, and reprocessed several times on their way to the inner disk. However, these models have not included internal viscous dissipation as an additional heating source. A warmer early disk can facilitate ice evolution and formation of complex organic molecules in the temperature regime between 20-40 K (Garrod et al. 2008; Nomura et al. 2009; Walsh et al. 2014; Drozdovskaya et al. 2014). Our results including the accretion heating indicate that significant ice reprocessing may occur out to larger radii than thought before, well in the comet formation zone. Such a reset scenario is supported by the meteoritic data from the inner few $\mathrm{AU}$, but the fraction of material that is reset at larger radii is still unknown (e.g., Pontoppidan et al. 2014). If some of the icy grains are incorporated into $\mathrm{km}$-sized bodies (planetesimals) immediately following disk formation, a larger fraction of the material could still remain pristine.

\section{Summary and conclusions}

Two-dimensional embedded disk models were presented to investigate the location of the snowlines of $\mathrm{H}_{2} \mathrm{O}, \mathrm{CO}_{2}$, and $\mathrm{CO}$. The dust temperature structure was calculated using the $3 \mathrm{D}$ dust radiative transfer code RADMC3D with a central heating source $\left(L_{\star}+L_{\mathrm{visc}}+L_{\mathrm{in}}\right)$. An additional heating term from an actively accreting disk was added through a diffusion approximation. The main parameters we explored here are $L_{\star}$, centrifugal radius, disk radius, disk mass, and stellar accretion rate. In addition, the extent of the snowline and optically thin water emission were compared with observations toward three deeply embedded lowmass YSOs. The following lists our main results.

- The presence of an envelope serves as a blanket for the disk such that the dust temperature within the inner few AU stays relatively warm for the adopted disk parameters. The adopted centrifugal radius affects the temperature structure of the disk since the effective midplane temperature depends on the optical depth. An envelope with a smaller centrifugal radius distributes more mass in the inner $100 \mathrm{AU}$ than an envelope with a higher centrifugal radius. Such a high concentration of mass in the inner $100 \mathrm{AU}$ increases the effective Rosseland mean optical depth that an observer at the disk midplane sees vertically, which in turn increases the effective midplane temperature. While it does not drastically affect the $T_{\text {dust }}>100 \mathrm{~K}$ regime, the presence of an envelope affects the location of $T_{\text {dust }} \lesssim 40 \mathrm{~K}$, which is important for the $\mathrm{CO}$ and $\mathrm{CO}_{2}$ snowlines.

- The midplane water snowline in the models can extend up to a maximum of $\sim 55 \mathrm{AU}$ for a disk accreting at $10^{-4} M_{\odot} \mathrm{yr}^{-1}$. The $\mathrm{CO}_{2}$ snowline is located at $\gtrsim 100$ AU for the same accretion rate. Both $\mathrm{H}_{2} \mathrm{O}$ and $\mathrm{CO}_{2}$ can remain in the solid phase at large radii $(R>100 \mathrm{AU})$ in the midplane within the boundaries of an embedded hydrostatic disk.

- CO is largely found to be in the gas phase within the embedded disk independent of accretion rate and disk properties. Some CO could be frozen out at large radii in the midplane, but only for a relatively massive embedded disk $\left(M_{\mathrm{d}} \geq 0.1 M_{\odot}\right)$.

- The $\mathrm{CO}_{2}$ and $\mathrm{CO}$ snowlines are affected by the exact structure of the flattened envelope. A smaller centrifugal radius corresponding to a highly flattened envelope model increases the $\mathrm{CO}$ and $\mathrm{CO}_{2}$ snow surfaces to larger radii.

- The observed $\mathrm{H}_{2}^{18} \mathrm{O}$ emission toward NGC 1333 IRAS 4A and IRAS 4B is consistent with models for $\dot{M}>$ $10^{-6} M_{\odot} \mathrm{yr}^{-1}$ for the adopted disk masses of $0.5 M_{\odot}$ and $0.2 M_{\odot}$, respectively. The observed size of water emission can also be explained with a model with a slightly higher accretion rate, but with a lower disk mass $(R<50 \mathrm{AU})$. Toward IRAS 2A, the models suggest a smaller disk contribution than toward IRAS 4A and IRAS 4B. Most of the optically thin water emission is emitted from the warm inner envelope (hot core) for that source (see Fig. 7).

- Significant chemical processing is expected to occur in the inner $100 \mathrm{AU}$ region during the disk formation process in 
stage 0. Midplane temperatures between 20-40 K are expected to be prevalent up to $100 \mathrm{AU}$ radius, and $100 \mathrm{~K}$ out to $30 \mathrm{AU}$, but only for the highest accretion rates found in the early phases of star formation. During these warm phases, the chemistry inherited from the collapsing cloud could be reset and more complex molecules could form, unless the ices are sequestered early into planetesimals.

In connection to early solar system formation, an important conclusion from our models is that a hot young solar nebula with water vapor out to $30 \mathrm{AU}$ can only occur during the deeply embedded phase, not in the T Tauri phase of our solar system. Our models also show that most of the observed optically thin water emission arises within the inner 50 AU radius. Future Atacama Large Millimeter/submillimeter Array (ALMA) observations spatially resolving the inner 50 AU can test our embedded accreting disk models when coupled with better physical models for individual sources. This will lead to better understanding of the physical and chemical structure and evolution of disks in the early stages of star formation.

Acknowledgements. We thank Atilla Juhász for providing the scripts for generating and analyzing RADMC3D input and output files. We also thank Kees Dullemond for providing RADMC3D. We are grateful to Catherine Walsh, Lee Hartmann, Joseph Mottram and Lars Kristensen for fruitful discussions. We are also grateful to the anonymous referee, whose comments have helped to improve the paper. This work is supported by the Netherlands Research School for Astronomy (NOVA). Astrochemistry in Leiden is supported by the Netherlands Research School for Astronomy (NOVA), by a Royal Netherlands Academy of Arts and Sciences (KNAW) professor prize, and by the European Union A-ERC grant 291141 CHEMPLAN. DH is funded by Deutsche Forschungsgemeinschaft Schwerpunktprogramm (DFG SPP 1385) The First 10 Million Years of the Solar System - a Planetary Materials Approach.

\section{References}

Aikawa, Y., Wakelam, V., Garrod, R. T., \& Herbst, E. 2008, ApJ, 674, 984 Andrews, S. M., Wilner, D. J., Hughes, A. M., Qi, C., \& Dullemond, C. P. 2009, ApJ, 700, 1502

Apai, D. A., \& Lauretta, D. S. 2010, Protoplanetary Dust: Astrophysical and Cosmochemical Perspectives (Cambridge: Cambridge Univ. Press)

Bachiller, R., \& Tafalla, M. 1999, in NATO ASIC Proc. 540: The Origin of Stars and Planetary Systems, eds. C. J. Lada, \& N. D. Kylafis, 227

Balbus, S. A., \& Hawley, J. F. 1998, Rev. Mod. Phys., 70, 1

Beckwith, S. V. W., Sargent, A. I., Chini, R. S., \& Guesten, R. 1990, AJ, 99, 924

Bisschop, S. E., Fraser, H. J., Öberg, K. I., van Dishoeck, E. F., \& Schlemmer, S. 2006, A\&A, 449, 1297

Bockelée-Morvan, D., Lis, D. C., Wink, J. E., et al. 2000, A\&A, 353, 1101 Brinch, C., Jørgensen, J. K., \& Hogerheijde, M. R. 2009, A\&A, 502, 199

Burke, D. J., \& Brown, W. A. 2010, Phys. Chem. Chem. Phys., 12, 5947

Calvet, N., Patino, A., Magris, G. C., \& D’Alessio, P. 1991, ApJ, 380, 617

Cassen, P. 2001, Meteorit. Planet. Sci., 36, 671

Chiang, E. I., \& Goldreich, P. 1997, ApJ, 490, 368

Ciesla, F. J., \& Cuzzi, J. N. 2006, Icarus, 181, 178

Cleeves, L. I., Bergin, E. A., Alexander, C. M. O. D., et al. 2014, Science, 345, 1590

Crapsi, A., van Dishoeck, E. F., Hogerheijde, M. R., Pontoppidan, K. M., \& Dullemond, C. P. 2008, A\&A, 486, 245

D’Alessio, P., Calvet, N., \& Hartmann, L. 1997, ApJ, 474, 397

D’Alessio, P., Cantö, J., Calvet, N., \& Lizano, S. 1998, ApJ, 500, 411

Davis, S. S. 2005, ApJ, 620, 994

Davis, A. M., Alexander, C. M. O., Ciesla, F. J., et al. 2014, in Protostars and Planets VI, eds. H. Beuther, C. Dullemond, \& T. Henning (Tucson: Univ. of Arizona Press), 809

de Graauw, T., Helmich, F. P., Phillips, T. G., et al. 2010, A\&A, 518, L6

Drozdovskaya, M. N., Walsh, C., Visser, R., Harsono, D., \& van Dishoeck, E. F. 2014, MNRAS, 445, 913

Dullemond, C. P., Hollenbach, D., Kamp, I., \& D’Alessio, P. 2007, in Protostars and Planets V, eds. B. Reipurth, D. Jewitt, \& K. Keil (Tucson: Univ. of Arizona Press), 555
Dunham, M. M., Stutz, A. M., Allen, L. E., et al. 2014, in Protostars and Planets VI, eds. H. Beuther, C. Dullemond, \& T. Henning (Tucson: Univ. of Arizona Press), 195

Fraser, H. J., Collings, M. P., McCoustra, M. R. S., \& Williams, D. A. 2001, MNRAS, 327, 1165

Gálvez, O., Ortega, I. K., Maté, B., et al. 2007, A\&A, 472, 691

Garaud, P., \& Lin, D. N. C. 2007, ApJ, 654, 606

Garrod, R. T., Weaver, S. L. W., \& Herbst, E. 2008, ApJ, 682, 283

Gibb, E. L., Whittet, D. C. B., Boogert, A. C. A., \& Tielens, A. G. G. M. 2004, ApJS, 151, 35

Grossman, L., \& Larimer, J. W. 1974, Rev. Geophys. Space Phys., 12, 71

Guilloteau, S., Piétu, V., Dutrey, A., \& Guélin, M. 2006, A\&A, 448, L5

Harsono, D., van Dishoeck, E. F., Bruderer, S., Li, Z.-Y., \& Jørgensen, J. K. 2015, A\&A, 577, A22

Hartmann, L., Calvet, N., Gullbring, E., \& D’Alessio, P. 1998, ApJ, 495, 385

Hasegawa, T. I., Herbst, E., \& Leung, C. M. 1992, ApJS, 82, 167

Herczeg, G. J., Karska, A., Bruderer, S., et al. 2012, A\&A, 540, A84

Hubeny, I. 1990, ApJ, 351, 632

Hueso, R., \& Guillot, T. 2005, A\&A, 442, 703

Jørgensen, J. K., \& van Dishoeck, E. F. 2010, ApJ, 710, L72

Jørgensen, J. K., Hogerheijde, M. R., Blake, G. A., et al. 2004, A\&A, 415, 1021

Jørgensen, J. K., Schöier, F. L., \& van Dishoeck, E. F. 2005, A\&A, 435, 177

Jørgensen, J. K., Bourke, T. L., Myers, P. C., et al. 2007, ApJ, 659, 479

Jørgensen, J. K., van Dishoeck, E. F., Visser, R., et al. 2009, A\&A, 507, 861

Karska, A., Herczeg, G. J., van Dishoeck, E. F., et al. 2013, A\&A, 552, A141

Kennedy, G. M., \& Kenyon, S. J. 2008, ApJ, 673, 502

Kerridge, J. F., \& Matthews, M. S. 1988, Meteorites and the early solar system (Tucson: Univ. of Arizona Press)

King, A. R., Pringle, J. E., \& Livio, M. 2007, MNRAS, 376, 1740

Kristensen, L. E., van Dishoeck, E. F., Bergin, E. A., et al. 2012, A\&A, 542, A8

Larson, R. B. 2003, Rep. Prog. Phys., 66, 1651

Lecar, M., Podolak, M., Sasselov, D., \& Chiang, E. 2006, ApJ, 640, 1115

Lewis, J. S. 1974, Science, 186, 440

Lewis, J. S., \& Prinn, R. G. 1980, ApJ, 238, 357

Li, Z.-Y., Banerjee, R., Pudritz, R. E., et al. 2014, in Protostars and Planets VI, eds. H. Beuther, C. Dullemond, \& T. Henning (Tucson: Univ. of Arizona Press), 173

Lissauer, J. J. 1987, Icarus, 69, 249

Lodato, G. 2008, New Astron. Rev., 52, 21

Lodders, K. 2004, ApJ, 611, 587

Lunine, J. I., Engel, S., Rizk, B., \& Horanyi, M. 1991, Icarus, 94, 333

Madhusudhan, N., Harrington, J., Stevenson, K. B., et al. 2011, Nature, 469, 64

Marboeuf, U., Thiabaud, A., Alibert, Y., Cabral, N., \& Benz, W. 2014, A\&A, 570, A36

Maret, S., Belloche, A., Maury, A. J., et al. 2014, A\&A, 563, L1

Mathews, G. S., Klaassen, P. D., Juhász, A., et al. 2013, A\&A, 557, A132

Meijerink, R., Pontoppidan, K. M., Blake, G. A., Poelman, D. R., \& Dullemond, C. P. 2009, ApJ, 704, 1471

Min, M., Dullemond, C. P., Dominik, C., de Koter, A., \& Hovenier, J. W. 2009, A\&A, 497, 155

Min, M., Dullemond, C. P., Kama, M., \& Dominik, C. 2011, Icarus, 212, 416

Moses, J. I., Line, M. R., Visscher, C., et al. 2013, ApJ, 777, 34

Mottram, J. C., van Dishoeck, E. F., Schmalzl, M., et al. 2013, A\&A, 558, A126

Mottram, J. C., Kristensen, L. E., van Dishoeck, E. F., et al. 2014, A\&A, 572, A21

Mousis, O., Lunine, J. I., Thomas, C., et al. 2009, ApJ, 691, 1780

Mousis, O., Lunine, J. I., Madhusudhan, N., \& Johnson, T. V. 2012, ApJ, 751, L7

Mumma, M. J., \& Charnley, S. B. 2011, ARA\&A, 49, 471

Murillo, N. M., Lai, S.-P., Bruderer, S., Harsono, D., \& van Dishoeck, E. F. 2013, A\&A, 560, A103

Nisini, B., Antoniucci, S., Giannini, T., \& Lorenzetti, D. 2005, A\&A, 429, 543

Nomura, H., Aikawa, Y., Nakagawa, Y., \& Millar, T. J. 2009, A\&A, 495, 183

Öberg, K. I., Boogert, A. C. A., Pontoppidan, K. M., et al. 2011a, ApJ, 740, 109

Öberg, K. I., Murray-Clay, R., \& Bergin, E. A. 2011b, ApJ, 743, L16

Ohashi, N., Saigo, K., Aso, Y., et al. 2014, ApJ, 796, 131

Owen, T., \& Bar-Nun, A. 1993, Nature, 361, 693

Persson, M. V., Jørgensen, J. K., \& van Dishoeck, E. F. 2012, A\&A, 541, A39

Persson, M. V., Jørgensen, J. K., \& van Dishoeck, E. F. 2013, A\&A, 549, L3

Persson, M. V., Jørgensen, J. K., van Dishoeck, E. F., \& Harsono, D. 2014, A\&A, 563, A74

Pollack, J. B., Hubickyj, O., Bodenheimer, P., et al. 1996, Icarus, 124, 62

Pontoppidan, K. M., Salyk, C., Bergin, E. A., et al. 2014, in Protostars and Planets VI, eds. H. Beuther, C. Dullemond, \& T. Henning (Tucson: Univ. of Arizona Press), 363

Pringle, J. E. 1981, ARA\&A, 19, 137

Qi, C., Wilner, D. J., Aikawa, Y., Blake, G. A., \& Hogerheijde, M. R. 2008, ApJ, 681,1396 
D. Harsono et al.: Volatile snowlines in embedded disks

Qi, C., Öberg, K. I., Wilner, D. J., et al. 2013, Science, 341, 630

Quanz, S. P., Amara, A., Meyer, M. R., et al. 2013, ApJ, 766, L1

Robitaille, T. P., Whitney, B. A., Indebetouw, R., Wood, K., \& Denzmore, P. 2006, ApJS, 167, 256

Ros, K., \& Johansen, A. 2013, A\&A, 552, A137

Rothman, L., Gordon, I., Barbe, A., et al. 2009, J. Quant. Spectr. Rad. Trans., 110,533

Sasselov, D. D., \& Lecar, M. 2000, ApJ, 528, 995

Scott, E. R. D. 2007, Ann. Rev. Earth Planet. Sci., 35, 577

Shakura, N. I., \& Sunyaev, R. A. 1973, A\&A, 24, 337

Stevenson, D. J., \& Lunine, J. I. 1988, Icarus, 75, 146

Testi, L., Birnstiel, T., Ricci, L., et al. 2014, in Protostars and Planets VI, eds. H. Beuther, C. Dullemond, \& T. Henning (Tucson: Univ. of Arizona Press), 339

Tobin, J. J., Hartmann, L., Chiang, H.-F., et al. 2012, Nature, 492, 83

Ulrich, R. K. 1976, ApJ, 210, 377

van der Tak, F. F. S., Walmsley, C. M., Herpin, F., \& Ceccarelli, C. 2006, A\&A, 447, 1011

van Dishoeck, E. F., Thi, W.-F., \& van Zadelhoff, G.-J. 2003, A\&A, 400, L1 van Dishoeck, E. F., Kristensen, L. E., Benz, A. O., et al. 2011, PASP, 123, 138 van Dishoeck, E. F., Bergin, E. A., Lis, D. C., \& Lunine, J. I. 2014, in Protostars and Planets VI, eds. H. Beuther, C. Dullemond, \& T. Henning (Tucson: Univ. of Arizona Press), 835

Visser, R., van Dishoeck, E. F., Doty, S. D., \& Dullemond, C. P. 2009, A\&A, 495,881

Visser, R., Doty, S. D., \& van Dishoeck, E. F. 2011, A\&A, 534, A132

Visser, R., Jørgensen, J. K., Kristensen, L. E., van Dishoeck, E. F., \& Bergin, E. A. 2013, ApJ, 769, 19

Vorobyov, E. I. 2009, ApJ, 704, 715

Walsh, C., Millar, T. J., \& Nomura, H. 2010, ApJ, 722, 1607

Walsh, C., Millar, T. J., Nomura, H., et al. 2014, A\&A, 563, A33

Wang, K.-S., van der Tak, F. F. S., \& Hogerheijde, M. R. 2012, A\&A, 543, A22

White, R. J., \& Hillenbrand, L. A. 2004, ApJ, 616, 998

Whitney, B. A., Wood, K., Bjorkman, J. E., \& Wolff, M. J. 2003, ApJ, 591, 1049

Williams, J. P., \& Cieza, L. A. 2011, ARA\&A, 49, 67

Wilson, T. L., \& Rood, R. 1994, ARA\&A, 32, 191

Zhang, K., Pontoppidan, K. M., Salyk, C., \& Blake, G. A. 2013, ApJ, 766, 82 


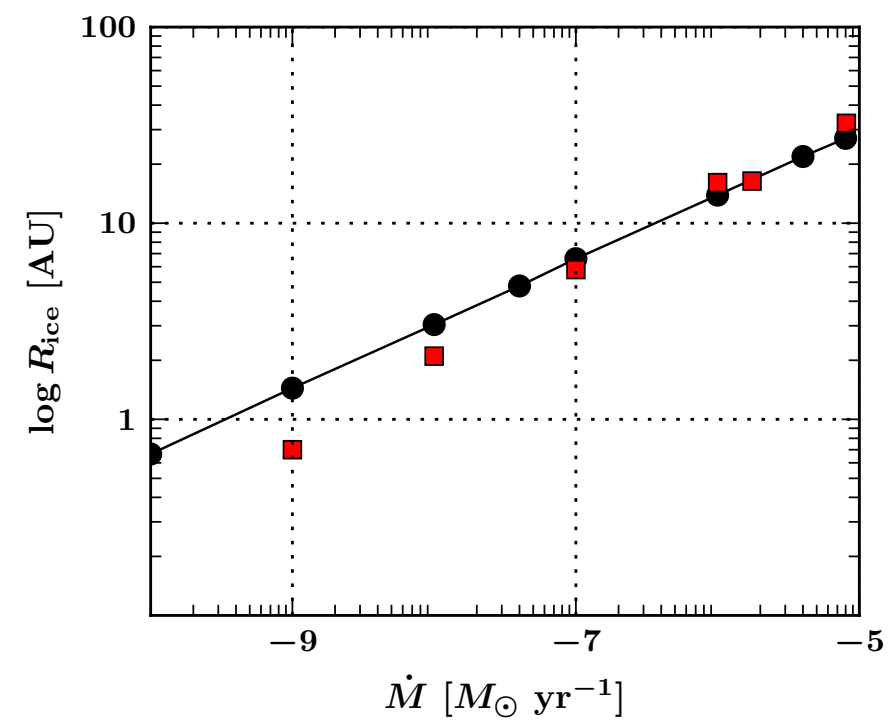

Fig. A.1. Snowlines for the MMSN disk without an envelope: black circles show the radii calculated with our method and red squares are tabulated values from Min et al. (2011).

\section{Appendix A: Snowline test}

Water snowlines were inferred and compared with results from Min et al. (2011) in Fig. A.1 using the minimum mass solar nebula (MMSN) model $\left(\Sigma \propto r^{-1.5}\right)$. The comparison shows that our adopted method reproduces the water snowlines at high accretion rates. For low accretion rates $\dot{M} \leq 10^{-9} M_{\odot} \mathrm{yr}^{-1}$, our values are slightly lower, but still consistent with those reported in literature.

\section{Appendix $\mathrm{B}: \mathrm{H}_{2} \mathrm{O}, \mathrm{CO}_{2}$, and $\mathrm{CO}$ gas fraction}

The $\mathrm{H}_{2} \mathrm{O}, \mathrm{CO}_{2}$ and $\mathrm{CO}$ gas pressure dependent gas fraction abundances are shown in Fig. B.1. Figures B.2 and B.3 show the midplane $\mathrm{CO}_{2}$ and $\mathrm{CO}$ snowlines as a function of luminosity and $R_{\mathrm{d}}$ similar to that of Fig. 9 for $\mathrm{H}_{2} \mathrm{O}$.
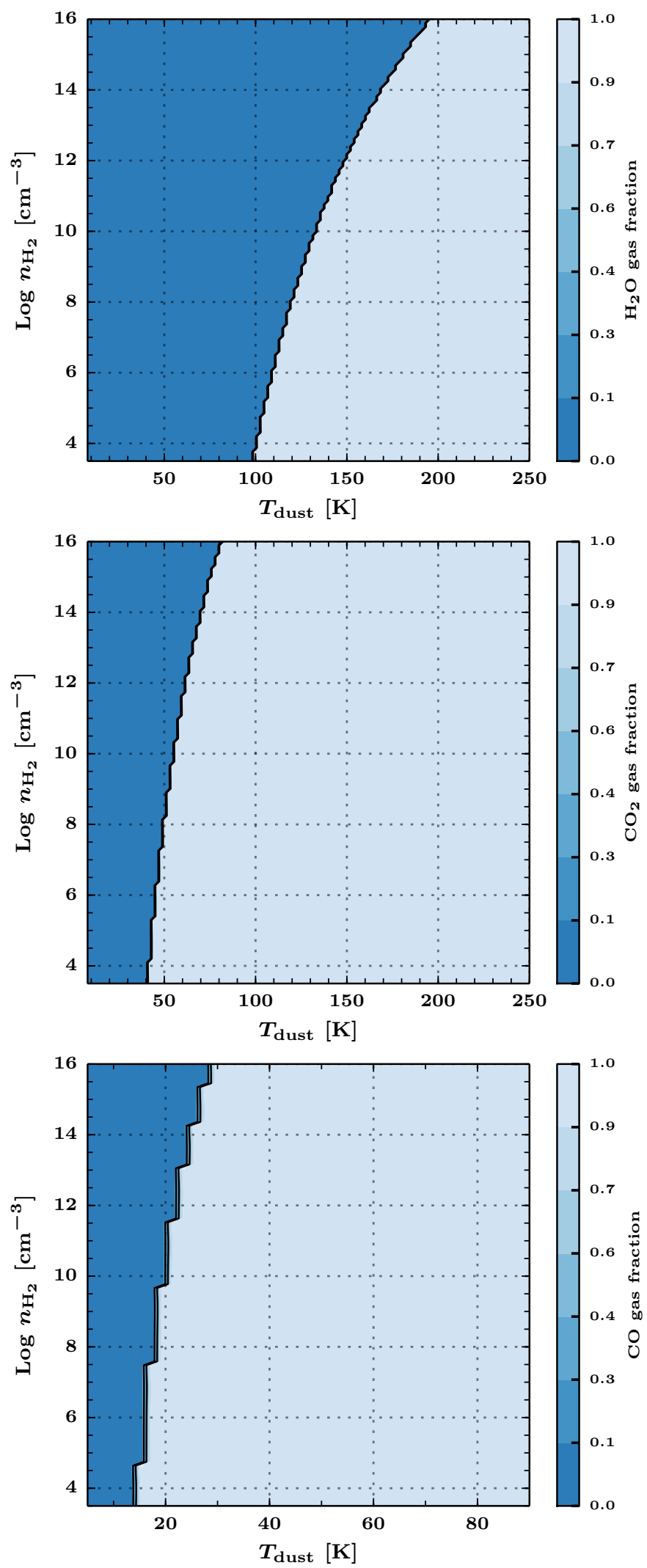

Fig. B.1. Gas fraction $\left(n_{\text {gas }} / n_{\text {gas }}+n_{\text {ice }}\right)$ for $\mathrm{H}_{2} \mathrm{O}(t o p), \mathrm{CO}_{2}$ (middle) and $\mathrm{CO}$ (bottom) as a function of density and temperatures. 
D. Harsono et al.: Volatile snowlines in embedded disks

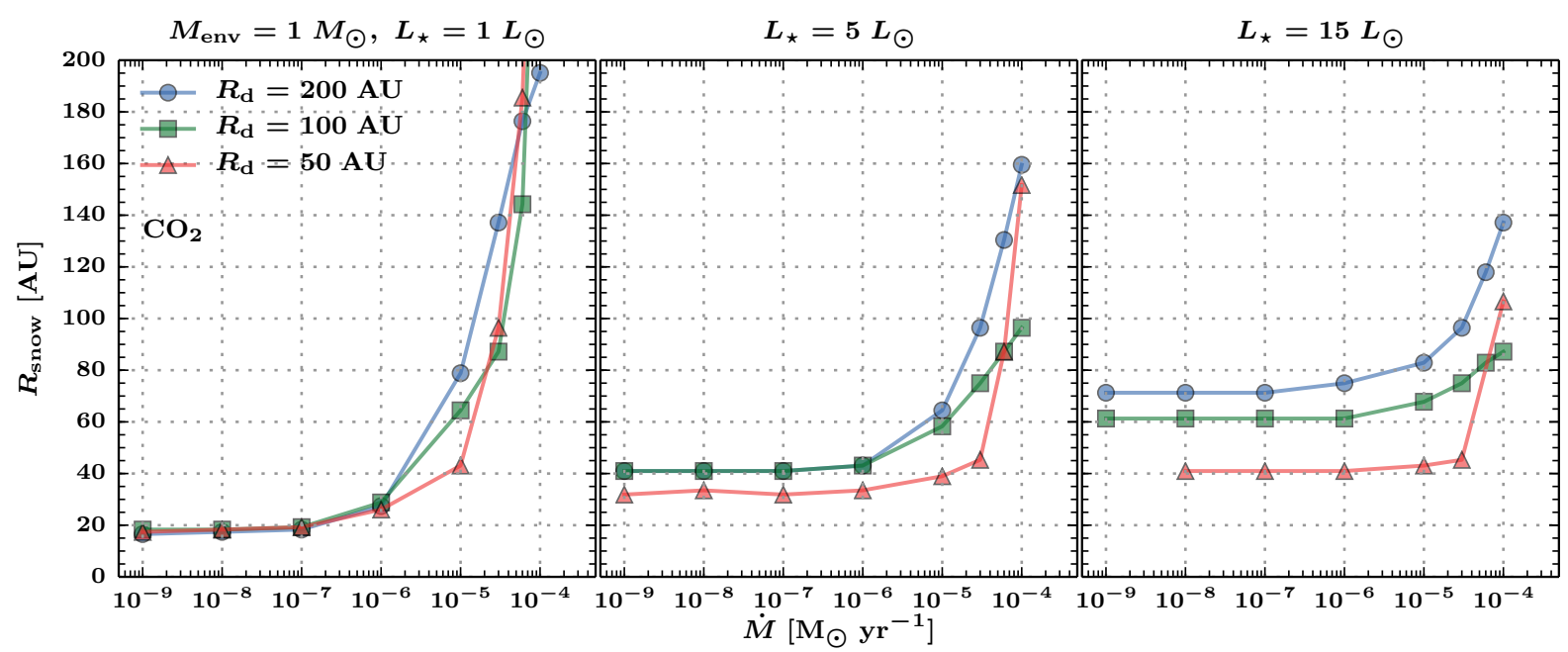

Fig. B.2. Midplane $\mathrm{CO}_{2}$ snowline as a function of stellar luminosity, accretion rate, and disk radius. The parameters are similar to that of Fig. 9 .

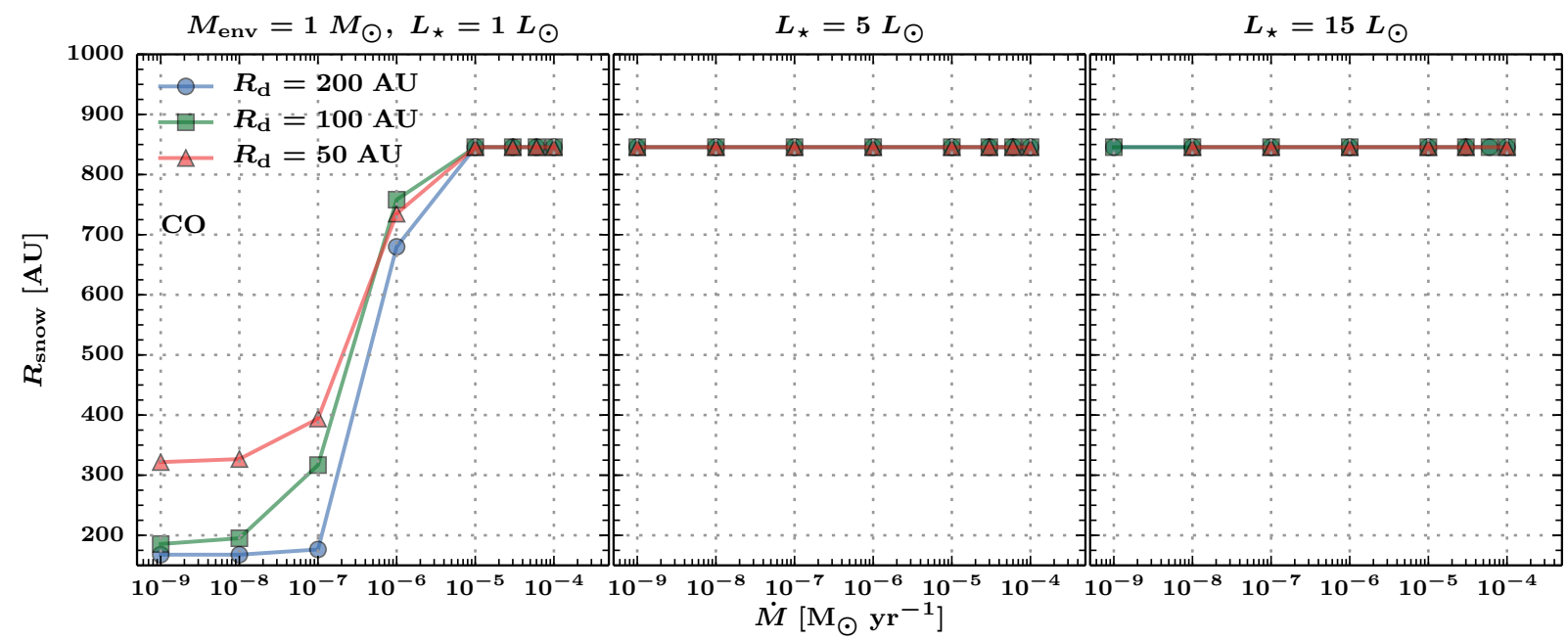

Fig. B.3. Midplane CO snowline as a function of stellar luminosity, accretion rate, and disk radius. The parameters are similar to that of Fig. 9. 